\title{
Homotopy groups of diagonal complements
}

\author{
SADOK KALLEL \\ INES SAIHI
}

For $X$ a connected finite simplicial complex we consider $\Delta^{d}(X, n)$, the space of configurations of $n$ ordered points of $X$ such that no $d+1$ of them are equal, and $B^{d}(X, n)$, the analogous space of configurations of unordered points. These reduce to the standard configuration spaces of distinct points when $d=1$. We describe the homotopy groups of $\Delta^{d}(X, n)$ (resp. $\left.B^{d}(X, n)\right)$ in terms of the homotopy (resp. homology) groups of $X$ through a range which is generally sharp. It is noteworthy that the fundamental group of the configuration space $B^{d}(X, n)$ abelianizes as soon as we allow points to collide, ie $d \geq 2$.

55Q52; 55P10

In memory of Abbas Bahri so greatly missed

\section{Introduction}

Let $X$ be a topological space and $\Delta_{d+1}(X, n) \subset X^{n}$ the union of the $(d+1)^{\text {st }}$ diagonal arrangement in $X^{n}$, that is,

$$
\begin{aligned}
\Delta_{d+1}(X, n)=\left\{\left(x_{1}, \ldots, x_{n}\right) \in X^{n} \mid x_{i_{0}}=\right. & x_{i_{1}}=\cdots=x_{i_{d}} \\
& \text { for some sequence } \left.1 \leq i_{0}<\cdots<i_{d} \leq n\right\} .
\end{aligned}
$$

Its complement in $X^{n}$ is the configuration space of no $d+1$ equal points in $X$, which is written

$$
\Delta^{d}(X, n)=X^{n}-\Delta_{d+1}(X, n) .
$$

This is the space of ordered tuples of $n$ points in $X$ with the multiplicity of each entry in the tuple at most $d$ (hence the notation $\Delta^{d}$ as opposed to $\Delta_{d}$ for at least $d$ ). It is useful to think of these tuples as configurations of $n$ ordered points in $X$ with the property that $d$ of the points can collide but not $d+1$. The symmetric group $\mathfrak{S}_{n}$ acts on $\Delta^{d}(X, n)$, and the quotient is denoted by $B^{d}(X, n)$. 
We have increasing filtrations

$$
\begin{aligned}
& F(X, n):=\Delta^{1}(X, n) \subset \Delta^{2}(X, n) \subset \cdots \subset \Delta^{n}(X, n)=X^{n}, \\
& B(X, n):=B^{1}(X, n) \subset B^{2}(X, n) \subset \cdots \subset B^{n}(X, n)=\operatorname{SP}^{n} X,
\end{aligned}
$$

with $\mathrm{SP}^{n} X:=X^{n} / \mathfrak{S}_{n}$ being the $n^{\text {th }}$ symmetric product. Here we have written $F(X, n)$ and $B(X, n)$ for the standard configuration spaces of ordered (resp. unordered) pairwise distinct points of cardinality $n$. Various other notations for $F(X, n)$ in the literature include $C_{n}(X), \operatorname{Conf}_{n}(X)$, etc, while $B(X, n)$ is sometimes written $\operatorname{Braid}(X, n)$ in the geometric topology literature; reminiscent of the fact that its fundamental group is the so-called $n^{\text {th }}$ braid group of $X$.

In some exceptional cases, the spaces $\Delta^{d}(X, n)$ and $B^{d}(X, n)$ can be empty (if, for example, $X$ is a point and $d<n)$, but otherwise they have a rich and interesting geometry; see Kallel and Taamallah [18]. An early appearance of $\Delta^{d}(X, n)$ is in paper of Cohen and Lusk [8] in connection with Borsuk-Ulam type results while more recent applications to the colored Tverberg theorem for manifolds appear in Blagojević, Matschke and Ziegler [4]. In the case $V$ is a vector space, the spaces $\Delta^{d}(V, n)$ are subspace complements dubbed non- $(d+1)$-equal arrangements in Björner and Welker [3], and their homology is made explicit in Dobrinskaya and Turchin [9] as an algebra over the little disks operad, with interesting applications to the spaces of non- $d$-equal immersions. In the case $X=\mathbb{C}$, the spaces $B^{d}(\mathbb{C}, n)$ are intimately related to spaces of based holomorphic maps from the Riemann sphere into complex projective space $\mathbb{P}^{d}$; see Guest, Kozlowski and Yamaguchi [12] and Kallel [16]. In all cases, these spaces seem to have been studied so far exclusively for when $X$ is a manifold. One of our objectives in this paper is to give some sharp results on the homology and homotopy groups of the non- $d$-equal configurations of $X$ when $X$ is a more general polyhedral space.

Throughout this paper, a space $X$ is a finite simplicial complex, that is, the realization of a finite abstract simplicial complex. Unless specified, all spaces are connected.

Theorem 1.1 Let $X$ be a connected finite simplicial complex that is not a point, and $d, n \geq 2$. Then

$$
\pi_{i}\left(B^{d}(X, n)\right) \cong \pi_{i}\left(\mathrm{SP}^{n}(X)\right) \text { for } 0 \leq i \leq 2 d-2 .
$$

In particular $\pi_{1}\left(B^{d}(X, n)\right) \cong H_{1}(X ; \mathbb{Z})$ when $d \geq 2, n \geq 2$. Moreover, if $X$ is simply connected, $2 \leq d \leq n$, then

$$
\pi_{i}\left(B^{d}(X, n)\right) \cong \tilde{H}_{i}(X ; \mathbb{Z}) \text { for } 0 \leq i \leq 2 d-2,
$$

where $\tilde{H}(-; \mathbb{Z})$ is reduced integral homology. 
The bound $2 d-2$ in the theorem is sharp as is illustrated by the case where $X$ a Euclidean space; see Section 4. Note that the special case of the fundamental group says that allowing a single collision is enough to abelianize the fundamental group. This can be expected since collisions kill the braiding; see Section 8.

The homotopy groups of $\Delta^{d}(X, n)$ turn out to depend on local connectivity properties of the space. We say $X$ has local homotopical dimension $r$ if for any $x \in X$ and any neighborhood $U$ of $x$, there is an open neighborhood $V \subset U$ of $x$ such that $V-\{x\}$ is $r$-connected; see Definition 7.1.

Theorem 1.2 Let $X$ be a locally finite simplicial complex with local homotopical dimension $r \geq 0, d \geq 1$. Then

$$
\pi_{i}\left(\Delta^{d}(X, n)\right) \cong \pi_{i}(X)^{n} \quad \text { for } i \leq r d+2 d-2 .
$$

Remark 1.3 If $d$ is at least $n$, both spaces are equal $\Delta^{d}(X, n)=X^{n}$ and all homotopy groups agree. When $d<n$ this bound is in general optimal as can be seen in the case of manifolds. For example, $\mathbb{R}^{2}$ has local homotopical dimension 0 and $\Delta^{d}\left(\mathbb{R}^{2}, d+1\right) \simeq$ $S^{2 d-1}$ is precisely $2 d-2-$ connected.

Remark 1.4 For a polyhedral pair $(X, Y)$, the homotopical depth of $Y$ in $X$ is set to be $n$ if the pair ( $X, X \backslash Y$ ) is $n$-connected; see Eyral [10]. Theorem 1.2 is saying that the homotopical depth of the diagonal arrangement $\Delta_{d+1}(X, n)$ in $X^{n}$ is at least $r d+2 d-2$. This appears to be the first complete such calculation for this kind of arrangements of subspaces.

To prove both of these theorems, we use a localization principle for homotopy groups, Theorem 4.2, relating the local connectivities of pairs $(V, V \backslash Y)$ to the global connectivity of $(X, X \backslash Y)$ for closed $Y \subset X$ and $V$ local neighborhoods in a cover. In both cases the proof reduces to studying the case of $V$ being the union of various simplices joining along a simplex. For Theorem 1.2, the argument amounts to giving a homotopical decomposition of $\Delta^{d}(V, n)$ when $V$ is such a union. We recall that by a homotopical decomposition of a space $X$ we mean a diagram $\mathcal{D}: I \rightarrow$ Top; ie a functor from a small category $I$ to the category of topological spaces and continuous maps, so that the map $\operatorname{hocolim}_{I} \mathcal{D} \rightarrow \operatorname{colim}_{I} \mathcal{D} \cong X$ is a weak equivalence; see Section 7. Our decomposition extends similar results of Sun [25]. Since we are able to control the connectivity of each space making up the diagram, we are able to derive our bound.

Theorem 1.1 on the other hand relies on a different argument. First we treat the case of a manifold based on the idea of scanning maps. The general case appeals to a theorem of Smale [24] relating the connectivity of a map to that of its preimages. 
Since Smale's theorem works for proper maps, a technical issue we have to deal with is the construction in Section 5 of a $\mathfrak{S}_{n}$-equivariant simplicial complex which is a deformation retract of $\Delta^{d}(X, n)$ for $X$ again a finite complex. As pointed out by the referee, similar techniques are in Björner et al [2, chapter 4] and have been applied to hyperplane arrangements by Blagojević and Ziegler [5], for example (see references therein). Section 5 is of independent interest and has relevance to more recent constructions of CW-retracts for configuration spaces; see Tamaki [26].

The first section of the paper discusses motivational examples and general connectivity results. The second section discusses the special case of graphs. Proposition 3.1 gives a simplified and then expanded version of a useful theorem of Morton, which is used to give an amusing description of the homotopy type of the configuration space of two points on a wedge of circles in Proposition 3.4.

Acknowledgment The first part of this work was conducted at the University of Lille 1 under a BQR grant. The Mediterranean Institute for the Mathematical Sciences (MIMS) has made resources available during the completion of this work. We are grateful to Faten Labassi for pointing us to Munkres' book and Proposition 5.1. Finally we thank Paolo Salvatore for his insight on Lemma 7.4.

\section{Preliminaries}

We start with some classical examples of diagonal arrangements and their complements. The extreme cases $d=1$ and $d=n-1$ are most encountered in the literature. The case $\Delta^{1}(X, n)=F(X, n)$ corresponds to the configuration space of pairwise distinct points

$$
F(X, n)=\left\{\left(x_{1}, \ldots, x_{n}\right) \in X^{n} \mid x_{i} \neq x_{j} \quad \text { for } i \neq j\right\} .
$$

The action of $\mathfrak{S}_{n}$ on $F(X, n)$ is free and we have a regular covering $F(X, n) \rightarrow$ $B(X, n)$. If $X$ is a manifold of dimension greater than 2 , then $\pi_{1}(F(X, n)) \cong \pi_{1}\left(X^{n}\right)$ by a codimension argument (see Proposition 2.5), while $\pi_{1}(B(X, n)$ ) is a wreath product $\pi_{1}(X) \prec \mathfrak{S}_{n}$; this is standard, but a leisurely exposition can be found in [15].

Example 2.1 When $d=n-1, B^{n-1}(X, n)$ is the complement in $\operatorname{SP}^{n}(X)$ of the diagonal embedding $\Delta: X \hookrightarrow \mathrm{SP}^{n} X, x \mapsto[x, \ldots, x]$. When $X=\mathbb{C}$, the elementary symmetric functions give a diffeomorphism $\operatorname{SP}^{n}(\mathbb{C}) \cong \mathbb{C}^{n}$ and the image of $\Delta(\mathbb{C})$ corresponds under this diffeomorphism to the rational normal curve $V$ diffeomorphic to the Veronese embedding $x \mapsto\left(x, x^{2}, \ldots, x^{n}\right)$. One can check that

$$
B^{n-1}(\mathbb{C}, n) \cong \mathrm{SP}^{n}(\mathbb{C})-V \simeq S^{2 n-3} \text {. }
$$


A short proof of this equivalence is given in [12, Lemma 2.7], while another quick argument would be to use simple connectivity of $B^{n-1}(\mathbb{C}, n)$ and Alexander duality. In general, for $\mathbb{R}^{k}, k \geq 2, \Delta^{n-1}\left(\mathbb{R}^{k}, n\right)=\left(\mathbb{R}^{k}\right)^{n}-\Delta$ is the complement of the thin diagonal, and this deforms onto the orthogonal complement of the diagonal $\Delta=\{(x, \ldots, x)\}$ minus the origin, so that $\Delta^{n-1}\left(\mathbb{R}^{k}, n\right)$ is, up to homotopy, the unit sphere $S^{n k-k-1}$ in $\left\{\left(x_{1}, \ldots, x_{n}\right) \in\left(\mathbb{R}^{k}\right)^{n} \mid \sum x_{i}=0\right\}=\Delta^{\perp}$. This deformation can be made equivariant with respect to the permutation action of $\mathfrak{S}_{n}$ so that the $\mathfrak{S}_{n}$-quotient is $B^{n-1}\left(\mathbb{R}^{k}, n\right)$. We show below that this space is simply connected as soon as $n$ is at least 3 (in fact it is $2 n-4$-connected; Lemma 4.12).

Lemma 2.2 If $S$ is the unit sphere in $H=\left\{\left(v_{1}, \ldots, v_{n}\right) \in\left(\mathbb{R}^{k}\right)^{n} \mid \sum v_{i}=0\right\}$, and if $\mathfrak{S}_{n}$ acts on $H$, and hence on $S$, by permutation of coordinates, then the quotient $Q_{n, k}:=S / \mathfrak{S}_{n}$ is simply connected whenever $n k-k-1 \geq 2$.

Proof We use the following useful main result of Armstrong [1]: let $G$ be a discontinuous group of homeomorphisms of a path connected, simply connected, locally compact metric space $X$, and let $H$ be the normal subgroup of $G$ generated by those elements that have fixed points; then the fundamental group of the orbit space $X / G$ is isomorphic to the factor group $G / H$. We apply this result to $G=\mathfrak{S}_{n}$ and $X=S$, which is simply connected. The point is that when $n \geq 3$, the fixed points of the permutation action are of the form $\left(v_{1}, \ldots, v_{n}\right)$ with $v_{i}=v_{j}$ for some $i<j$, which means that all transpositions are in $H$ and hence $G=H$.

The argument of Armstong used in the proof of Lemma 2.2 implies that if $\Delta^{d}(X, n)$ is simply connected, then $\pi_{1}\left(B^{d}(X, n)\right)$ is the quotient of $\mathfrak{S}_{n}$ by the normal subgroup generated by elements having fixed points, and this subgroup is the entire group if $d \geq 2$. This establishes a useful conclusion.

Corollary 2.3 If $\Delta^{d}(X, n)$ is simply connected, then so is $B^{d}(X, n)$ if $d \geq 2$.

The following result, valid for smooth manifolds, is a special case of Theorem 1.1.

Proposition 2.4 When $X=M$ is a closed smooth, ie $C^{\infty}$, manifold, $\operatorname{dim} M \geq 2$, and $n \geq 3$, then $\pi_{1}\left(B^{n-1}(M, n)\right)$ is isomorphic to $H_{1}(M ; \mathbb{Z})$.

Proof A tubular neighborhood of the diagonal copy of $M$ in $\mathrm{SP}^{n} M$ can be identified with the total space of the following subbundle. Let $T M^{\oplus n}$ be the $n$-fold Whitney sum of the tangent bundle $T M$ of $M, \operatorname{dim} M=m$, and let $\eta$ be the subbundle with fiber $H=\left\{\left(v_{1}, \ldots, v_{n}\right) \mid \sum v_{i}=0\right\}$. The total space of this subbundle is homeomorphic to a neighborhood of diagonal $M$ in $M^{\times n}$. Now $\mathfrak{S}_{n}$ acts on this bundle fiberwise (linearly on each fiber) and the fiberwise quotient $\zeta$ has fiber $H / \mathfrak{S}_{n}$ which can be identified with the cone $c\left(S^{(n-1) m-1} / \mathfrak{S}_{n}\right)$, where $\operatorname{dim} M=m$ and $S^{(n-1) m-1}$ is the 
unit sphere in $H$. According to [18, Proposition 4.1], for a smooth closed manifold $M$, a neighborhood deformation retract $V$ of the diagonal $M$ in $\mathrm{SP}^{n} M$ is homeomorphic to the total space of $\zeta$. The fiberwise apexes of the fiberwise cone give the zero section of this bundle. The complement of this section is $S(M)$ which is, up to fiberwise equivalence, a bundle over $M$ with fiber $S^{(n-1) m-1} / \mathfrak{S}_{n}$. By construction we have the homotopy pushout

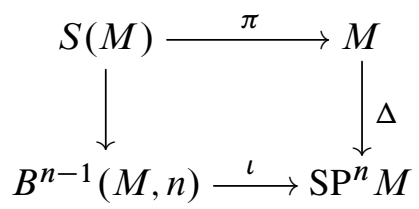

If $n=2, m \geq 2, S(M)$ is the projectivized tangent bundle with fiber $S^{m-1} / \mathbb{Z}_{2}=$ $\mathbb{R} P^{m-1}$. When $n \geq 3$ and $m \geq 2, S(M)$ has simply connected fiber (Lemma 2.2) so that $\pi$ induces an isomorphism on fundamental groups, and by the van Kampen theorem, $\iota$ induces an isomorphism on $\pi_{1}$ as well; ie $\pi_{1}\left(B^{n-1}(M, n)\right) \cong \pi_{1}\left(\mathrm{SP}^{n} M\right) \cong$ $H_{1}(M ; \mathbb{Z})$ for $n \geq 3$.

To complete this section, we state a well-known result which will be seen in Section 4 as a special manifestation of the localization principle.

Proposition 2.5 If $S=\bigcup S_{j}$ is a finite union of submanifolds of a smooth manifold $M$, closed with real codimension $d \geq 2$, then the inclusion $M-S \hookrightarrow M$ induces an isomorphism on homotopy groups $\pi_{i}$ for $0 \leq i \leq d-2$, and an epimorphism on $\pi_{d-1}$.

A proof of the above proposition, using standard transversality arguments, can be found, for example, in [14, Lemma 5.3]. This proposition is not true if the ambient space is not a manifold. For example, $B\left(\mathbb{R}^{m}, 2\right)$ is the complement of the diagonal in $\operatorname{SP}^{2}\left(\mathbb{R}^{m}\right)$ and we have the homotopy equivalence $B\left(\mathbb{R}^{m}, 2\right) \simeq \mathbb{R} P^{m-1}$ so that $\pi_{1}\left(B\left(\mathbb{R}^{m}, 2\right)\right) \cong \mathbb{Z}_{2}$ no matter the codimension of the diagonal $m \geq 3$.

As a consequence we have the following precursor of Theorem 1.1.

Corollary 2.6 If $X$ is a topological surface and $d \geq 2$, then $\pi_{1}\left(B^{d}(X, n)\right) \cong H_{1}(X, \mathbb{Z})$.

Proof The real plane $\mathbb{R}^{2}$ has the special property that $\mathrm{SP}^{n}\left(\mathbb{R}^{2}\right)$ is diffeomorphic to $\mathbb{R}^{2 n}$. This implies right away that when $S$ is a topological surface, $\operatorname{SP}^{n}(S)$ is a manifold of dimension $2 n$, and that $B_{d+1}(X, n)$ is the union of submanifolds of dimension at most $2(n-d)=2 n-2 d$. This means that $B^{d}(S, n)=\operatorname{SP}^{n}(S)-$ $B_{d+1}(S, n)$ is the complement of a finite union of submanifolds of codimension at least $2 d>2$. By Proposition 2.5, $\pi_{1}\left(B^{d}(S, n)\right) \cong \pi_{1}\left(\mathrm{SP}^{n} S\right)$ and this is again $H_{1}(S, \mathbb{Z})$ for $n>1$. 


\section{The case of the circle}

Write $S^{1}=\{z \in \mathbb{C}|| z \mid=1\}$, ie the unit circle in the complex plane. There is a map $B^{d}\left(S^{1}, n\right) \rightarrow S^{1}$ which multiplies the points of a configuration in $S^{1}$. This map is well defined since $S^{1}$ is abelian. This map turns out to have contractible fibers so that in particular $B^{d}\left(S^{1}, n\right) \simeq S^{1}$; see Proposition 3.2.

Let $\Delta_{n-1}=\left\{\left(s_{1}, \ldots, s_{n}\right) \mid 0 \leq s_{i} \leq 1, \sum s_{i}=1\right\}$ be the $n-1$-dimensional simplex and write $\Delta_{n-1}(d)$ the partial compactification of the open simplex $\stackrel{\circ}{\Delta}_{n-1}$, where we allow at most $d$ consecutive $s_{i}$ to be zero (using cyclic ordering, ie $s_{n}$ and $s_{1}$ are consecutive to each other). In particular $\Delta_{n}(1)=\stackrel{\circ}{\Delta}_{n-1}$. We will write $\mathbb{Z}_{n}$ for the cyclic group of order $n$. Using a similar action as in [6, page 407], we have the following.

Proposition 3.1 Let $\mathbb{Z}_{n}$ with multiplicative generator $\tau$ act on $S^{1} \times \Delta_{n-1}(d)$ via

$$
\tau\left(e^{i \theta}, s_{1}, \ldots, s_{n}\right)=\left(e^{i \theta+i 2 \pi s_{1}}, s_{2}, \ldots, s_{n}, s_{1}\right) .
$$

Then the quotient by the action, written $S^{1} \ltimes_{\mathbb{Z}_{n}} \Delta_{n-1}(d)$, is homeomorphic to $B^{d}\left(S^{1}, n\right)$. When $d=1$, there is a $\mathfrak{S}_{n}$-equivariant homeomorphism

$$
F\left(S^{1}, n\right) \cong\left(S^{1} \times \stackrel{\circ}{\Delta}_{n-1}\right) \times_{\mathbb{Z}_{n}} \mathfrak{S}_{n} .
$$

Proof The cyclic group appears for a simple reason: any configuration $\left(x_{1}, \ldots, x_{n}\right)$ can be brought into a unique counterclockwise configuration up to cyclic permutation. More precisely let $\left(x_{1}, \ldots, x_{n}\right) \in \Delta^{d}\left(S^{1}, n\right)$. Then there is a permutation $\sigma \in \mathfrak{S}_{n}$ bringing this configuration to a counterclockwise ordering $\left(x_{\sigma(1)}, \ldots, x_{\sigma(n)}\right)$. Let $s_{i}$ be the arc distance (divided by $2 \pi$ ) measured counterclockwise between $x_{\sigma(i)}$ and $x_{\sigma(i+1)}$. When $x_{i} \neq x_{j}$ for $i \neq j$, the choice of $\sigma$ is unique up to cyclic permutation and there is a well-defined map

$$
\begin{aligned}
F\left(S^{1}, n\right) & \rightarrow\left(S^{1} \times \stackrel{\circ}{\Delta}_{n-1}\right) \times_{\mathbb{Z}_{n}} \mathfrak{S}_{n}, \\
\left(x_{1}, \ldots, x_{n}\right) & \mapsto\left[\left(x_{\sigma(1)},\left(s_{1}, \ldots, s_{n}\right)\right) ; \sigma\right],
\end{aligned}
$$

which is a homeomorphism. Here $\left(s_{1}, \ldots, s_{n}\right)$ is in the open simplex $\stackrel{\circ}{\Delta}_{n-1}$ if and only if none of the $s_{i}$ are zero. When there is collision, ie $d>1$, then the choice of $\sigma$, up to cyclic permutation, is not unique anymore, but there is a map at the level of unordered configuration spaces

$$
\begin{aligned}
B^{d}\left(S^{1}, n\right) & \rightarrow S^{1} \ltimes_{\mathbb{Z}_{n}} \Delta_{n-1}(d), \\
{\left[x_{1}, \ldots, x_{n}\right] } & \mapsto\left[x_{\sigma(1)} ;\left(s_{1}, \ldots, s_{n}\right)\right],
\end{aligned}
$$

where $\sigma$ again is any permutation bringing $\left(x_{1}, \ldots, x_{n}\right)$ into cyclic ordering. 
This map is independent of the choice of $\sigma$ and it is a homeomorphism with inverse $\left[x_{\sigma(1)} ;\left(s_{1}, \ldots, s_{n}\right)\right] \mapsto\left[x_{\sigma(1)}, x_{\sigma(1)} e^{i 2 \pi s_{1}}, x_{\sigma(1)} e^{i 2 \pi\left(s_{1}+s_{2}\right)}, \ldots, x_{\sigma(1)} e^{i 2 \pi\left(s_{1}+\cdots+s_{n-1}\right)}\right]$. Note that when $x_{i}=x_{i+1}$ in the cyclic ordering, $s_{i}=0$, so the faces of $\Delta_{n-1}$ where the $s_{i}$ vanish (consecutively) correspond to when points come together.

Proposition 3.2 Identify $S^{1}=[0,1] / 0 \sim 1$. Then addition

$$
m: B^{d}\left(S^{1}, n\right) \rightarrow S^{1}, \quad m\left(\left[x_{1}, \ldots, x_{n}\right]\right)=x_{1}+x_{2}+\cdots+x_{n}
$$

is a bundle map with fiber $\Delta_{n-1}(d)$. In particular $m$ is a homotopy equivalence.

Proof The composite

$$
\rho: S^{1} \times_{\mathbb{Z}_{n}} \Delta_{n-1}(d) \rightarrow B^{d}\left(S^{1}, n\right) \stackrel{m}{\longrightarrow} S^{1}
$$

sends $\left(x,\left(s_{1}, \ldots, s_{n}\right)\right)$ to $n x+(n-1) s_{1}+(n-2) s_{2}+\cdots+s_{n-1}$. This map is well defined on orbits since $\rho\left(x+s_{1},\left(s_{2}, \ldots, s_{n}, s_{1}\right)\right)=\rho\left(x,\left(s_{1}, \ldots, s_{n}\right)\right)$. The preimage of a point $y \in S^{1}$ under $m$ are all unordered tuples $\left[x_{1}, \ldots, x_{n}\right]$ such that $x_{1}+x_{2}+\cdots+x_{n}=y \bmod \mathbb{Z}$. All preimages are homeomorphic and we can choose $y=0$. The preimage $\rho^{-1}(0)$ consists of all classes $\left[x,\left(s_{1}, \ldots, s_{n}\right)\right]$ such that

$$
(n-1) s_{1}+(n-2) s_{2}+\cdots+s_{n-1}+n x \quad \bmod \mathbb{Z} .
$$

We wish to show this is a copy of $\Delta_{n-1}(d)$. Consider the map $\phi: \Delta_{n-1}(0) \rightarrow \rho^{-1}(0)$ defined as follows. Given $\left(s_{1}, \ldots, s_{n}\right), \sum s_{i}=1$, let

$$
m_{s}=\frac{-1}{n}\left((n-1) s_{1}+(n-2) s_{2}+\cdots+s_{n-1}\right)
$$

brought modulo $\mathbb{Z}$ to the interval $[0,1]$ and define

$$
\phi:\left(s_{1}, \ldots, s_{n}\right) \mapsto\left[m_{s},\left(s_{1}, \ldots, s_{n}\right)\right] \in S^{1} \times_{\mathbb{Z}_{n}} \Delta_{n-1}(d) .
$$

This map is well defined and continuous. It is surjective by construction. It is also injective for the following reason. If $s=\left(s_{1}, \ldots, s_{n}\right)$ and $s^{\prime}=\left(s_{1}^{\prime}, \ldots, s_{n}^{\prime}\right)$ map to the same point under $\phi$, they must be the same up to cyclic permutation. Let's assume $s^{\prime}=\left(s_{k+1}, \ldots, s_{n}, s_{1}, s_{2}, \ldots, s_{k}\right), 0<k<n\left(s_{0}=s_{n}\right)$. A quick computation shows that

$$
m_{s^{\prime}}=m_{s}+s_{1}+\cdots+s_{k}-k / n .
$$

But in $S^{1} \times_{\mathbb{Z}_{n}} \Delta_{n-1}(d),\left[m_{s^{\prime}},\left(s_{1}^{\prime}, \ldots, s_{n}^{\prime}\right)\right]=\left[m_{s}-k / n,\left(s_{1}, \ldots, s_{n}\right)\right]$ so that $\phi\left(s^{\prime}\right)$ can never be $\phi(s)$ unless $k=0$ or $s_{i}=s_{i}^{\prime}=1 / n$. In both cases $s=s^{\prime}$. This proves the injectivity and hence that $\phi$ is a homeomorphism. It remains to check that $\rho$ is a bundle map and this is left as an exercise. 
Remark 3.3 (Morton) When $d=1, m: \operatorname{SP}^{n}\left(S^{1}\right) \rightarrow S^{1}$ is an $n$-1-disk bundle that is trivial if and only if $n$ is odd. The open disk bundle is $B\left(S^{1}, n\right)$ and its sphere bundle is $B_{2}\left(S^{1}, n\right)$.

\section{A Wedges of circles}

As discussed, $B^{d}\left(S^{1}, n\right) \simeq S^{1}$. The situation gets more complicated quickly for other graphs. The following is a neat little application of our constructions for the case $d=1$.

Proposition 3.4 $B\left(\bigvee^{k} S^{1}, 2\right)$ is homotopy equivalent to $\bigvee^{\frac{3}{2} k(k-1)+1} S^{1}$.

Proof Let's first understand the $k=2$ case.

We will write $B\left(S^{1} \vee S^{1}, 2\right)$ as the union of three subspaces:

$$
\begin{gathered}
X_{1}=\{[(x, *),(y, *)] \mid x \neq y\}, \quad X_{2}=\{[(*, x),(*, y)] \mid x \neq y\}, \\
X_{3}=\{[(x, *),(*, y)] \mid(x, y) \neq(*, *)\} .
\end{gathered}
$$

We have that

$$
X_{1} \cong B\left(S^{1}, 2\right), \quad X_{2} \cong B\left(S^{1}, 2\right), \quad X_{3} \cong\left(S^{1} \times S^{1}\right)^{*},
$$

where $\left(S^{1} \times S^{1}\right)^{*}$ means the punctured torus $S^{1} \times S^{1}-\{(*, *)\}$. Notice that $X_{1} \cap$ $X_{2}=\varnothing$ while $X_{1} \cap X_{3}=\{(x, *),(*, *), x \neq *\} \cong\left(S^{1}\right)^{*}$ are punctured circles hence contractible intervals. The punctured torus $X_{3}$ deformation retracts onto a wedge $S^{1} \vee S^{1}$. During this deformation both punctured circles corresponding to the intersection with $X_{1}$ and $X_{2}$ retract onto the wedgepoint. After the retraction we obtain a wedge $S^{1} \vee S^{1} \vee Y_{1} \vee Y_{2}$ where each $Y_{i}=X_{i} / \sim$ is the open Möbius band $\left.X_{i}=S^{1} \times\right] 0,1[$ with an interval $* \times] 0,1\left[\right.$ retracted to a point. Therefore $Y_{i} \simeq S^{1}$ and the claim follows in this case.

For the general case of a bouquet of $k$-circles, $k>2$, we write an element from the $i^{\text {th }}$ leaf as $x^{i}$. Then $B\left(\bigvee^{k} S^{1}, 2\right)$ becomes the union of subspaces

$$
\begin{aligned}
X_{i, j} & :=\left\{\left[\left(x^{i}, *\right),\left(y^{j}, *\right)\right] \mid x^{i} \neq y^{j} \text { if } i=j\right\}, \\
X_{i}^{j} & :=\left\{\left[\left(x^{i}, *\right),\left(*, y^{j}\right)\right] \mid\left(x^{i}, y^{j}\right) \neq(*, *) \text { if } i=j\right\}, \\
X^{i, j} & :=\left\{\left[\left(*, x^{i}\right),\left(*, y^{j}\right)\right] \mid x^{i} \neq y^{j} \quad \text { if } i=j\right\},
\end{aligned}
$$

over all $k \geq i \geq j \geq 1$. As before $X_{i, i}=B\left(S^{1}, 2\right)$ is the open Möbius band. For $i>j, X_{i, i}$ and $X_{j, j}$ are disjoint. Also and as is clear, $X_{i}^{j} \cap X_{r}^{s}=\varnothing$ if $\{i, j\} \neq\{r, s\}$. Each union $B_{i, j}:=X_{i, j} \cup X_{i, i} \cup X_{i}^{j}$ is the subconfiguration space of 2 points on the $i^{\text {th }}$ and $j^{\text {th }}$ leaves and hence is, up to homotopy, a wedge of 4 circles. The homotopy 

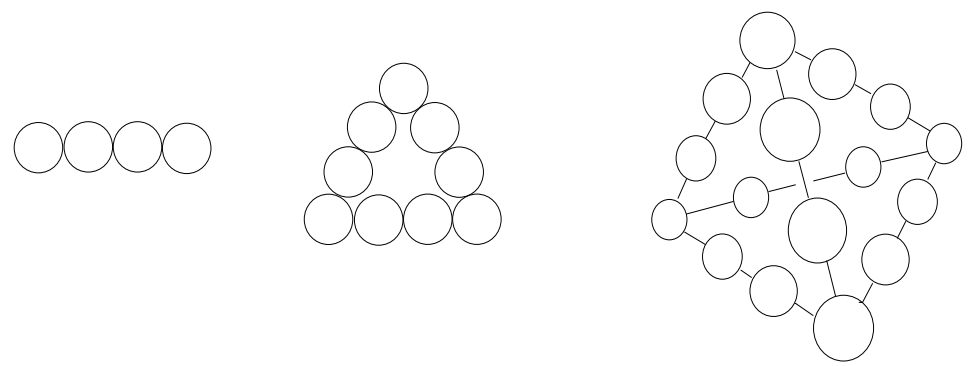

Figure 1: An intermediate homotopy type of $B\left(\bigvee^{k} S^{1}, 2\right)$ for $k=2,3$ and 4 , respectively. These are strings of circles making up a necklace in the shape of a $k-1$-dimensional simplex.

deforming each $X_{i, i}$ to $S^{1}$ is the same if performed in $B_{i, j}$ or $B_{i, k}$. This is to say that the homotopies deforming the $B_{i, j}$ to a wedge of 4 circles are compatible and we obtain a deformation retract of $B\left(\bigvee^{k} S^{1}, 2\right)$ which looks like a necklace of circles tied in the shape of the $k-1$-dimensional simplex. This is depicted in Figure 1 for $k=2,3$ and 4 .

The homotopy type of this space is not hard to work out: it is a wedge of all those circles appearing in the necklace with another wedge of circles describing the homotopy type of the 1-skeleton of $\Delta_{k-1}$. In the necklace there is one circle for each vertex of the $k-1-$ simplex and two circles for each edge, this gives a total of $k^{2}$ circles. On the other hand the one-skeleton of the $k-1$-simplex, denoted by $\Delta_{k-1}^{(1)}$, is homotopy equivalent to $\bigvee^{N} S^{1}$ where $N=\frac{1}{2} k(k-3)+1$ circles. Indeed the Euler characteristic

$$
\chi\left(\Delta_{k-1}^{(1)}\right)=\text { \#edges }-\# \text { vertices }=\frac{1}{2} k(k-1)-k=\frac{1}{2} k(k-3),
$$

and this must be $\chi\left(\bigvee^{N} S^{1}\right)=N-1$. Putting this together yields

$$
B\left(\bigvee^{k} S^{1}, 2\right) \simeq \bigvee^{k^{2}} S^{1} \bigvee^{\frac{1}{2} k(k-3)+1} S^{1} \simeq \bigvee^{\frac{3}{2} k(k-1)+1} S^{1}
$$

and the proof is complete.

Remark 3.5 The first homology group of $B(\Gamma, n)$ for graphs has been worked out in [21]. Their method uses discrete Morse theory. In particular one can deduce from [21, Theorem 3.16] that $H_{1}\left(B\left(\bigvee^{k} S^{1}, 2\right)\right)=\mathbb{Z}^{1+3 k(k-1) / 2}$ in full agreement with our Proposition 3.4 (in their theorem one uses that the braid index is $2, N_{1}=$ $2 k(k-1)-\frac{1}{2} k(k-1)-(k-1)$ and the first Betti number of the graph is of course $\left.k\right)$. In the case of trees $T$, the homology groups of the unordered configuration space $B(T, n)$ are torsion free and their ranks computed by Farley; references and details are in [21]. 


\section{The localization principle and the case of manifolds}

Our main approach is to find conditions on $X$ so that the inclusion $B^{d}(X, n) \hookrightarrow \operatorname{SP}^{n}(X)$ induces an isomorphism on some homotopy groups through a range. We start with a preliminary lemma. We say a space $X$ is locally punctured connected if for every $x \in X$ and neighborhood $U$ of $x$, there is an open $V, x \in V \subset U$ such that $V-\{x\}$ is connected.

Lemma 4.1 Let $X$ be path-connected, locally contractible, and not a point. If $d \geq 2$, then both $\Delta^{d}(X, n)$ and $B^{d}(X, n)$ are connected. If, furthermore, $X$ is locally punctured connected, then both $\Delta^{d}(X, n)$ and $B^{d}(X, n)$ are connected for all $d \geq 1$.

Proof For both claims, it suffices to show that $\Delta^{d}(X, n)$ is connected. We need to join $\left(x_{1}, \ldots, x_{n}\right)$ to $\left(y_{1}, \ldots, y_{n}\right)$ by a path, for any two choices of tuples in $\Delta^{d}(X, n)$. By deforming locally, we can arrange that the $x_{i}$ and the $y_{j}$ are all pairwise distinct. Now $X$ is path-connected so there is a path $\gamma_{i}$ from $x_{i}$ to $y_{i}$. Via $\gamma_{1}$ we construct a path in $\Delta^{d}(X, n)$ from $\left(x_{1}, x_{2}, \ldots, x_{n}\right)$ to $\left(y_{1}, x_{2}, \ldots, x_{n}\right)$ by putting $\gamma_{1}(t)$ in the first coordinate. At any given time $t \in[0,1], \gamma_{1}(t)$ can only coincide with one $x_{i}$ at a time, and hence this path is well defined in $\Delta^{d}(X, n)$ if $d \geq 2$. Construct next the path from $\left(y_{1}, x_{2}, \ldots, x_{n}\right)$ to $\left(y_{1}, y_{2}, x_{3}, \ldots, x_{n}\right)$ by putting $\gamma_{2}(t)$ in the second coordinate. This is again a well-defined path in $\Delta^{d}(X, n)$. We can continue this process. The composition $\gamma_{n} \circ \cdots \circ \gamma_{1}$ is a path in $\Delta^{d}(X, n)$ from $\left(x_{1}, \ldots, x_{n}\right)$ to $\left(y_{1}, \ldots, y_{n}\right)$.

To establish the second claim, we proceed by induction on $n \geq d$. For $n=d$, $\Delta^{d}(X, d)=X^{d}$ and there is nothing to prove. For $n>d$, consider the projection

$$
\Delta^{d}(X, n) \rightarrow \Delta^{d}(X, n-1)
$$

that omits the last coordinate. The preimage of a tuple $\left(x_{1}, \ldots, x_{n-1}\right)$ is $X-$ $\left\{x_{i_{1}}, \ldots, x_{i_{j}}\right\}$ if $x_{i_{r}}$ repeats $d$-times in the tuple. Since $X$ is locally punctured connected, this preimage is connected. Since the base space of this projection is also connected by inductive hypothesis, it follows that the total space is connected, as desired.

For the higher homotopy groups, the starting point is the following principle, which relates the local connectivity properties of a space to its global properties. All spaces appearing below are connected. The following result is in [20, Theorem 1.4].

Theorem 4.2 (localization) Let $X$ be a Hausdorff topological space and $Y$ be a closed subset of $X$. If for every point $y \in Y$, and every neighborhood $U \subset X$ of $y$, there is an open $V \subset U$ containing $y$ such that the pair $(V, V \backslash Y)$ is $k$-connected, $k \geq 0$, then the pair $(X, X \backslash Y)$ is $k$-connected. 
We recall what it means for the pair $(X, A)$ to be $k$-connected or that $\pi_{i}(X, A)=0$ for all $i \leq k$ [13, Chapter 4]. If $k \geq 1$, this means that every map $\left(I^{r}, \partial I^{r}\right) \rightarrow(X, A)$ from the closed cube $I^{r}, 1 \leq r \leq k$, is homotopic (relative its boundary) to a map $I^{r} \rightarrow A$. For any $x \in X,(X,\{x\})$ is 1 -connected if and only if $X$ is simply connected. Being 0 -connected, or equivalently writing $\pi_{0}(X, A)=0$, means in our terms that $X$ and $A$ are connected and that any point in $X$ is connected by a path to a point in $A$. Note that in the theorem, if either $V \backslash Y$ or $X \backslash Y$ is not connected, then the theorem fails.

Example 4.3 $X=\mathbb{R}^{3}$ and $L$ a line in $\mathbb{R}^{3}$. The pair $(X, X \backslash L)$ is 1 -connected but not 2-connected. Indeed take a square which is intersected transversally through its interior by $L$. That square cannot be deformed away from $L$ with the boundary being kept fixed.

The following is a consequence of Theorem 4.2. We say that a closed subset $Y$ in $X$ is tame if there is a neighborhood $N$ of $Y$ such that $N$ deformation retracts onto $Y$ and $X \backslash Y$ deformation retracts onto $X \backslash N$. Submanifolds are tame and so are subcomplexes of simplicial complexes; see Proposition 5.1.

Corollary 4.4 Let $Y$ be a tame subspace of $X$ and suppose for every $y \in Y$ and neighborhood $U$ of $y$ in $X$, there is a contractible neighborhood $V \subset U$, such that $Y \cap V$ is tame in $V$ and $V \backslash Y$ is $k$-connected, $k \geq 0$. Then $\pi_{i}(X) \cong \pi_{i}(X \backslash Y)$ for $i \leq k$.

Proof The point is that when $Y$ is tame in $X$, Theorem 4.2 implies that the induced map $\pi_{k}(X \backslash Y) \rightarrow \pi_{k}(X)$ is surjective, and $\pi_{i}(X \backslash Y) \rightarrow \pi_{i}(X)$ is an isomorphism for $i \leq k-1$. Let's show that for $(V, y)$ as in the statement of the theorem, the pair $(V, V \backslash Y)$ is $k+1$-connected. Since $V \cap Y$ is tame in $V$, choose a neighborhood $N$ of $Y$ in $V$ that deformation retracts onto $Y$ and such that $V \backslash N$ deformation retracts onto $V \backslash Y$. We can replace, up to homotopy, the pair $(V, V \backslash Y)$ by $(V, V \backslash N)$, where now $V \backslash N$ is closed in $V$. We can apply the long exact sequence in homotopy of the pair $(V, V \backslash N)$

$$
\begin{aligned}
\rightarrow \pi_{k+1} V \rightarrow \pi_{k+1}(V, V \backslash N) \stackrel{\partial}{\longrightarrow} \pi_{k}(V \backslash N) & \rightarrow \cdots \\
& \rightarrow \pi_{1}(V, V \backslash N) \stackrel{\partial}{\longrightarrow} \pi_{0}(V \backslash N) \rightarrow \pi_{0}(V) .
\end{aligned}
$$

Since for $i \leq k, \pi_{i}(V \backslash N)=0=\pi_{i+1}(V)$, we see that $\pi_{i}(V, V \backslash Y) \cong \pi_{i}(V, V \backslash N)=0$ for $i \leq k+1$. From Theorem 4.2 it follows that $(X, X \backslash Y)$ is $k+1$-connected. The same argument as above with the long exact sequence of the pair $(X, Y)$ with $Y$ tame in $X$ shows that $\pi_{i}(X) \cong \pi_{i}(X \backslash Y)$ for $i \leq k$. 
Remark 4.5 In the case of a submanifold $S$ in $M$ of codimension $d$, a neighborhood of a point deformation retracts onto a sphere $S^{d-1}$, which is $d-2-$ connected. By the previous corollary this gives that $M$ is weakly equivalent to $M-S$ up to dimension $d-2$ (Proposition 2.5). A similar argument applies when $S=\bigcup S_{j}$ is the union of submanifolds intersecting transversally.

The following key lemma shows how we can apply the above results to diagonal arrangements.

Lemma 4.6 Let $X$ be a finite simplicial complex such that for every $x \in X$ and neighborhood $U$ of $x$, there is a subneighborhood $V$ containing $x$ such that $\Delta^{d}(V, k)$ (resp. $\left.B^{d}(V, k)\right)$ is $r$-connected for any $k \geq 1$. Then $\pi_{i}\left(\Delta^{d}(X, n)\right) \cong \pi_{i}\left(X^{n}\right)$ (resp. $\left.\pi_{i}\left(\operatorname{SP}^{n} X\right) \cong \pi_{i}\left(B^{d}(X, n)\right)\right)$ for $i \leq r$.

Proof We have to estimate the connectivity of the pair $\left(X^{n}, \Delta^{d}(X, n)\right)=\left(X^{n}, X^{n}-\right.$ $\left.\Delta_{d+1}(X, n)\right)$ (resp. that of $\left(\operatorname{SP}^{n} M, \operatorname{SP}^{n}(M)-B_{d+1}(M, n)\right)$. Note that $\Delta_{d+1}(M, n)$ (resp. $\left.B_{d+1}(M, n)\right)$ is tame in $M^{n}$ (resp. $\mathrm{SP}^{n} M$ ) according to Section 5. One can check they verify the hypothesis of Corollary 4.4. In the ordered case, choose a point in $\Delta_{d+1}(X, n)$ which, after permutation, can be brought to the form

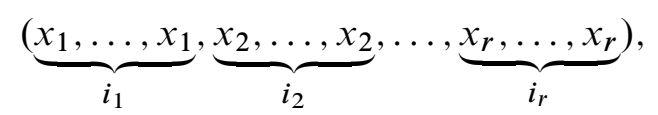

with $x_{i} \neq x_{j}$ if $i \neq j, \sum i_{\alpha}=n$ and $i_{1}>d$. A neighborhood $W$ of this point in $X^{n}$ is homeomorphic to $V_{1}^{i_{1}} \times \cdots \times V_{r}^{i_{r}}$, where $V_{i}$ is a contractible neighborhood of $x_{i}$ in $X$, and the $V_{i}$ are pairwise disjoint. Clearly

$$
W-\Delta_{d+1}(X, n) \cong \Delta^{d}\left(V_{1}, i_{1}\right) \times \cdots \times \Delta^{d}\left(V_{r}, i_{r}\right) .
$$

By hypothesis we can assume all the $\Delta^{d}\left(V_{i}, i_{j}\right)$ to be $r$-connected so that $W-$ $\Delta_{d+1}(X, n)$ is also $r$-connected and hence, by Corollary $4.4, \pi_{i}\left(\Delta^{d}(X, n)\right)=$ $\pi_{i}\left(X^{n}-\Delta_{d+1}(X, n)\right) \cong \pi_{i}\left(X^{n}\right)$ for $i \leq r$.

A similar proof holds in the unordered case. Given a point in $B_{d+1}(M, n) \subset \operatorname{SP}^{n}(M)$ as in (2), a small contractible neighborhood of it in $\mathrm{SP}^{n} M$ is

$$
U \cong \operatorname{SP}^{i_{1}}\left(V_{1}\right) \times \operatorname{SP}^{i_{2}}\left(V_{2}\right) \times \cdots \times \mathrm{SP}^{i_{r}}\left(V_{r}\right)
$$

the $V_{i}$ are pairwise distinct, and

$$
B^{d}(U, n)=U-B_{d+1}(X, n) \cong B^{d}\left(V_{1}, i_{1}\right) \times B^{d}\left(V_{2}, i_{2}\right) \times \cdots \times B^{d}\left(V_{r}, i_{r}\right) .
$$

If we choose each $V_{j}$ so that $B^{d}\left(V_{j}, i_{j}\right)$ is $r$-connected (hypothesis), the complement $B^{d}(U, n)$ will also be $r$-connected and the claim follows again from Corollary 4.4. 
In the case of a manifold, we can already make the following easy conclusions.

Corollary 4.7 Let $M$ be a manifold of dimension $m \geq 1$.

(i) If $m d \geq 3$, then $\pi_{i}\left(\Delta^{d}(M, n)\right) \cong \pi_{i}(M)^{n}$ for $i \leq m d-2$.

(ii) If $m \geq 2$ and $d \geq 2$, then $\pi_{1}\left(B^{d}(M, n)\right) \cong H_{1}(M, \mathbb{Z})$.

Proof Every point of $M$ has a neighborhood homeomorphic to $\mathbb{R}^{m}$. The fat diagonal $\Delta_{d+1}\left(\mathbb{R}^{m}, n\right)$ in $\left(\mathbb{R}^{m}\right)^{n}$ has codimension $m n-m(n-d)=m d \geq 3$, so its complement $\Delta^{d}\left(\mathbb{R}^{m}, n\right)$ is $m d-2-$ connected (Proposition 2.5). Now apply Lemma 4.6 to get (i). On the other hand $\Delta^{d}\left(\mathbb{R}^{m}, k\right)$ is simply connected if $d \geq 2$ and $m \geq 2$, so by Armstrong's result (Corollary 2.3), $\pi_{1}\left(B^{d}\left(\mathbb{R}^{m}, k\right)\right)$ is also trivial and (ii) follows.

Remark 4.8 As we pointed out, Corollary 4.7(i) is not true for $m d=2$ as illustrated by $F\left(\mathbb{R}^{2}, 2\right) \simeq S^{1}$. This corollary is a special case of Theorem 1.1 . Also let's point out that $\Delta^{d}\left(\mathbb{R}^{m}, n\right)$ has torsion free homology starting with spherical classes in $d m-1$ as already indicated, and all homology classes are represented by products of spheres [9].

We now derive Theorem 1.1 when $X$ is a manifold. Again $X$ is $r$-connected if $\pi_{i}(X)=0$ for $0 \leq i \leq r$.

Lemma 4.9 Let $\Omega_{*}^{m}(-)$ denote a connected component of the loop space $\Omega^{m}(-)$, $m \geq 1$ and $d \geq 1$. Then $\Omega_{*}^{m} \mathrm{SP}^{d} S^{m}$ is $2 d-2-$ connected.

Proof Let's review the simplest cases. The case $d=1$ is obvious since $\Omega^{m} S^{m}$ breaks down into components indexed by the integers, and each component is $0-$ connected but not 1 -connected since $\pi_{1}\left(\Omega_{*}^{m} S^{m}\right) \cong \pi_{m+1}\left(S^{m}\right)$ is $\mathbb{Z}$ if $m=2$ and $\mathbb{Z}_{2}$ if $m \geq 3$. When $m=1, \mathrm{SP}^{d} S^{1} \simeq S^{1}$ so that $\Omega_{*} S^{1}$ is contractible and hence certainly $2 d-2-$ connected for any $d$. When $m=2, \mathrm{SP}^{d} S^{2} \cong \mathbb{P}^{d}$ is complex projective space and

$$
\Omega^{2} \mathrm{SP}^{d} S^{2}=\Omega^{2} \mathbb{P}^{d} \cong \mathbb{Z} \times \Omega^{2} S^{2 d+1}
$$

Each component is a copy of $\Omega^{2} S^{2 d+1}$, which is $2 d-2-$ connected, and the bound is sharp.

In general we invoke [18, Theorem 5.9] which states that for $r$-connected $X, r \geq 1$,

$$
\pi_{i}\left(\mathrm{SP}^{n} X\right) \cong \tilde{H}_{i}(X ; \mathbb{Z}), \quad 0 \leq i \leq r+2 n-1 .
$$

This gives that for $i \geq 1$ and $m \geq 2$,

$$
\begin{gathered}
\pi_{i}\left(\Omega_{*}^{m} \mathrm{SP}^{d} S^{m}\right) \cong \pi_{i+m}\left(\mathrm{SP}^{d} S^{m}\right) \cong H_{i+m}\left(S^{m}\right)=0, \\
i+m \leq(m-1)+2 d-1=m+2 d-2 .
\end{gathered}
$$

This gives $i \leq 2 d-2$ and a lower bound for the connectivity is $2 d-2$. 
Proposition 4.10 Assume $m \geq 2, n \geq d \geq 1$. Then $B^{d}\left(\mathbb{R}^{m}, n\right)$ is $2 d-2$-connected. Moreover if $X$ is a 1 -connected manifold and $n \geq 2$, then $\pi_{i}\left(B^{d}(X, n)\right) \cong \widetilde{H}_{i}(X ; \mathbb{Z})$ for $0 \leq i \leq 2 d-2$.

Proof This relies on results from [16; 18]. The case $d=1$ being trivial, we assume $d \geq 2$. Consider the sequence of embeddings

$$
\begin{aligned}
\tau_{n}: B^{d}\left(\mathbb{R}^{m}, n\right) & \hookrightarrow B^{d}\left(\mathbb{R}^{m}, n+1\right), \\
{\left[x_{1}, \ldots, x_{n}\right] } & \mapsto\left[x_{1}, \ldots, x_{n},\left|x_{1}\right|+\cdots+\left|x_{n}\right|+1\right] .
\end{aligned}
$$

The direct limit is $B^{d}\left(\mathbb{R}^{m}, \infty\right)$, and it is shown in [16] that there is a scanning map

$$
\tau: B^{d}\left(\mathbb{R}^{m}, \infty\right) \rightarrow \Omega_{*}^{m} \mathrm{SP}^{d} S^{m}
$$

that induces a homology isomorphism. Since both spaces are simply connected when $d \geq 2$ (Corollary 2.3 and Lemma 4.9) and have the homotopy type of CW complexes, the map $\tau$ is a homotopy equivalence. Moreover, the maps $\tau_{n}$ in (5) induce homology embeddings according to [27, Chapitre 3]. Iterating, we get homology embeddings

$$
H_{*}\left(B^{d}\left(\mathbb{R}^{m}, d+1\right)\right) \hookrightarrow H_{*}\left(B^{d}\left(\mathbb{R}^{m}, n\right) \hookrightarrow H_{*}\left(B^{d}\left(\mathbb{R}^{m}, \infty\right)\right) \cong H_{*}\left(\Omega_{*}^{m} \operatorname{SP}^{d} S^{m}\right) .\right.
$$

By Lemma 4.9 the groups on the extreme right are trivial for $* \leq 2 d-2$. This gives that $H_{*}\left(B^{d}\left(\mathbb{R}^{m}, n\right)\right)=0$ for $n \geq d$ and $* \leq 2 d-2$. Since the space is simply connected, it is $2 d-2$-connected as well. It then follows by Lemma 4.6 that $\pi_{i}\left(B^{d}(X, n)\right) \cong \pi_{i}\left(\mathrm{SP}^{n}(X)\right)$ for $i \leq 2 d-2$. This proves the main statement. In the case $X$ is $r$-connected with $r \geq 1$, it follows by the inequality in (4), since $2 d-2 \leq r+2 n-1$, that $\pi_{i}\left(B^{d}(X, n)\right) \cong \pi_{i}\left(\operatorname{SP}^{n}(X)\right) \cong \tilde{H}_{i}(X ; \mathbb{Z})$ in the range of dimensions $0 \leq i \leq 2 d-2$.

Example 4.11 Consider the case $B^{n-1}\left(S^{2}, n\right), n \geq 3$. Since $\operatorname{SP}^{n}\left(S^{2}\right) \cong \mathbb{P}^{n}$ is a $2 n$-dimensional manifold, by Proposition $2.5, \pi_{i}\left(B^{n-1}\left(S^{2}, n\right)\right) \cong \pi_{i}\left(\mathbb{P}^{n}\right)$ for $1 \leq i \leq$ $2(n-1)-2=2 n-4$. On the other hand, from the Hopf fibration, $\pi_{i}\left(\mathbb{P}^{n}\right) \cong \pi_{i}\left(S^{2 n+1}\right)$ for $i>2$ and $\pi_{2}\left(\mathbb{P}^{n}\right)=\mathbb{Z}$. This shows precisely that $\pi_{i}\left(B^{n-1}\left(S^{2}, n\right)\right) \cong H_{i}\left(S^{2}, \mathbb{Z}\right)$ for $1 \leq i \leq 2 n-4$, as expected.

The claim that $B^{d}\left(\mathbb{R}^{k}, n\right)$ is $2 d-2-$ connected has an nice alternative proof in the case $d=n-1$.

Lemma 4.12 $B^{n-1}\left(\mathbb{R}^{k}, n\right)$ is $2 n-4-$ connected, $n \geq 2, k \geq 1$.

Proof The case $k=1$ is trivial. We let $k \geq 2$ and invoke some main results from [17; 18]. Let $S$ be the unit sphere as in Lemma 2.2 and let $Q_{n, k}$ be its quotient under the 
$\mathfrak{S}_{n}$-action. We have already indicated that $Q_{n, k} \simeq B^{n-1}\left(\mathbb{R}^{k}, n\right)$. On the other hand, according to [17, Theorems 1.1, 1.3 and 1.5],

$$
\Sigma^{k+1} Q_{n, k} \simeq \overline{\mathrm{SP}}^{n}\left(S^{k}\right),
$$

where $\Sigma$ means suspension and $\overline{\mathrm{SP}}^{n}(Y)$ means the symmetric smash $Y^{\wedge(n)} / \mathfrak{S}_{n}$, which is also the cofiber of the embedding of $\mathrm{SP}^{n-1} Y$ into $\mathrm{SP}^{n} Y$ induced by adjoining a basepoint to an unordered tuple $\left[x_{1}, \ldots, x_{n-1}\right]$. It is shown [17, Theorems 1.2 and 1.3] that if $X$ is $r$-connected, then $\overline{\mathrm{SP}}^{n}(\Sigma X)$ is $2 n+r-1$-connected. This gives that $\overline{\mathrm{SP}}^{n}\left(S^{k}\right)=\overline{\mathrm{SP}}^{n}\left(\Sigma S^{k-1}\right)$ is $2 n+k-3$-connected, and hence so is $\Sigma^{k+1} Q_{n, k}$ by (6). Since in this range $Q_{n, k}$ is already simply connected, it must therefore be $2 n-4-$ connected.

Remark 4.13 That the connectivity bound in the above theorem doesn't depend on $k$ is not surprising. Indeed when $n=2, B\left(\mathbb{R}^{k}, 2\right) \simeq \mathbb{R} P^{k-1}$ and this is never 1 -connected no matter what $k$ is.

\section{An equivariant deformation retract of diagonal complements}

Let $X_{*}$ be an abstract simplicial complex and $\left|X_{*}\right|$ its geometric realization. Let $A_{*}$ be a subcomplex of $X_{*}$. We say a subcomplex $A_{*}$ of $X_{*}$ is full if every simplex of $X_{*}$ whose vertices are in $A_{*}$ is itself in $A_{*}$. The following fundamental result (called the retraction lemma in [5]) can be found in Munkres' book [22, Lemma 70.1].

Proposition 5.1 Let $A_{*}$ be a full subcomplex of the finite simplicial complex $X_{*}$. Let $C_{*}$ consist of all simplices of $X_{*}$ that are disjoint from $A_{*}$. Then $\left|A_{*}\right|$ is a deformation retract of $\left|X_{*}\right|-\left|C_{*}\right|$, and $\left|C_{*}\right|$ is a deformation retract of $\left|X_{*}\right|-\left|A_{*}\right|$.

The argument of proof is short but instrumental to extract useful properties of this compactification. We review this argument. The fact that $A_{*}$ is full says that $C_{*}$ is also full, and that simplices of $X_{*}$ consist of simplices in $C_{*}$, simplices in $A_{*}$ and simplices of the form

$$
\sigma * \tau, \quad \sigma \in A_{*}, \quad \tau \in C_{*},
$$

where $\sigma * \tau$ is the join of both simplices. Figure 2 illustrates the situation when $X_{*}$ is the full simplex $\Delta_{3}$ on 4 vertices $v_{0}, v_{1}, v_{2}, v_{3}, A_{*}=\left[v_{0} v_{1}\right]$ and $C_{*}=\left[v_{2} v_{3}\right]$.

The deformation of $\left|X_{*}\right|-\left|A_{*}\right|$ onto $\left|C_{*}\right|$ is as in the figure. It starts at a point $t x+\sum_{i \in I} s_{i} v_{i}$, with $v_{i}$ vertices in $C_{*}, i \in I, t+\sum s_{i}=1, t \neq 1$, and ends at the point $\sum\left(t_{j} / \sum s_{i}\right) v_{j}, j \in I$. 

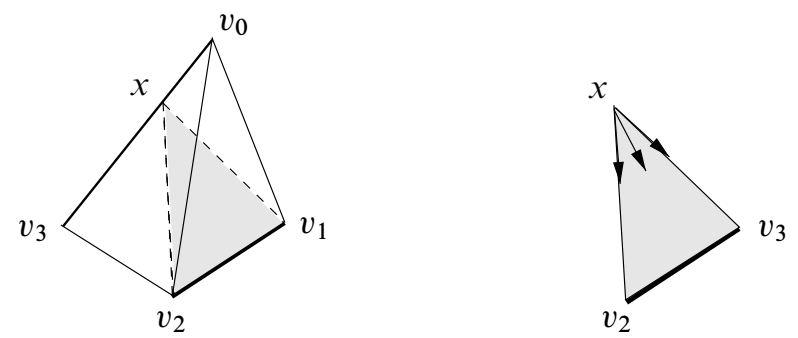

Figure 2: Munkres' deformation along the join (right) after deleting the apex $x$

Two important consequences are in order:

- If $A_{*}$ is full, $\left|X_{*}\right|-\left|A_{*}\right|$ deformation retracts onto the largest subcomplex that does not meet $\left|A_{*}\right|$. Note that if $A_{*}$ is not full, then its first barycentric subdivision $\mathrm{Sd} A_{*}$ is always full in $\mathrm{Sd} X_{*}$. The barycentric subdivision comes with a natural ordering on vertices.

- The deformation retraction illustrated in Figure 2 has the property that if it starts in a simplex of $X_{*}$ it will stay in that simplex (and deforms onto a face of it).

For ease we will write $X$ for either $X_{*}$ or its realization. The context will be clear.

Munkres' observation nicely applies to the diagonal arrangements. Given $X$ an ordered simplicial complex, $X^{n}$ can be given naturally a structure of a simplicial complex such that the various diagonals are subcomplexes; see [23, Section 1], and also the proof of Lemma 5.2 below. We can then apply Proposition 5.1 to the configuration space $X^{n}-\Delta_{d+1}(X, n)$. Among all diagonal arrangements, only the thin diagonal $\Delta_{n}(X, n)$ is full. We therefore have to pass to a barycentric subdivision. Let $\operatorname{Sd}\left(X^{n}\right)$ be the barycentric subdivision of $X_{*}^{n}$. This restricts to $\operatorname{Sd}\left(\Delta_{d+1}(X, n)\right)$.

Lemma 5.2 There is an $\mathfrak{S}_{n}$-equivariant deformation retraction of $\Delta^{d}(X, n)$ onto the largest subcomplex $W^{d}(X, n)$ not intersecting $\left|\operatorname{Sd}\left(X^{n}\right)\right|-\left|\operatorname{Sd}\left(\Delta_{d+1}(X, n)\right)\right|$.

Proof That the complement deformation retracts onto $W^{d}(X, n)$ is a direct consequence of Proposition 5.1 as applied to the pair $\left(\operatorname{Sd}\left(X^{n}\right), \operatorname{Sd}\left(\Delta_{d+1}(X, n)\right)\right)$ with $\operatorname{Sd}\left(\Delta_{d+1}(X, n)\right)$ being full. We need check this deformation is equivariant under the symmetric group action. Recall that the simplicial decomposition of $X^{n}$ is made out as follows, where $X$ of course is an ordered simplicial complex [23]. A vertex of $X^{n}$ is of the form $\left(v_{1}, \ldots, v_{n}\right)$ where $v_{i}$ is a vertex of $X$. Different $(q+1)$-vertices

$$
w_{0}=\left(v_{01}, \ldots, v_{0 n}\right), \quad w_{1}=\left(v_{11}, \ldots, v_{1 n}\right), \quad \ldots, \quad w_{q}=\left(v_{q 1}, \ldots, v_{q n}\right),
$$

form a $q$-dimensional simplex if and only if for each $k=1,2, \ldots, n,(q+1)$-vertices $v_{0 k}, v_{1 k}, \ldots, v_{q k}$ are contained in a simplex of $X$ and $v_{0 k} \leq v_{1 k} \leq \cdots \leq v_{q k}$; see Figure 3 for the decomposition of $X^{3}$ in the case $X=[0,1]$ with vertices $[0] \leq[1]$. 

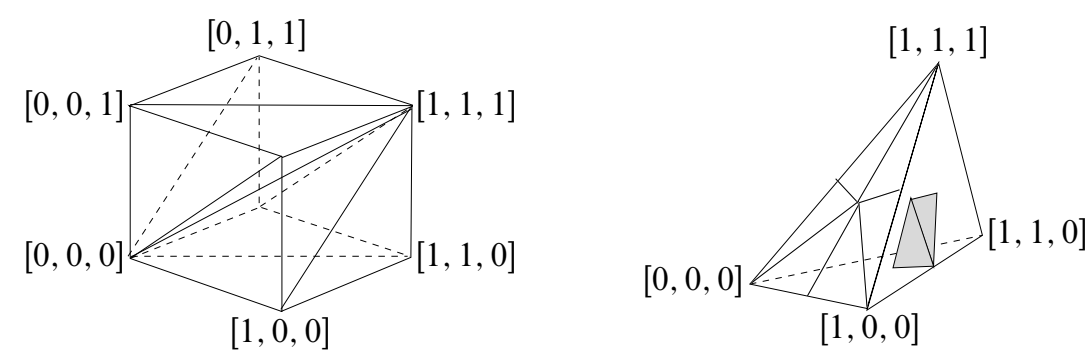

Figure 3: Left: simplicial decomposition for $[0,1]^{3}$ with 8 vertices, 19 edges, 18 triangular faces and 6 tetrahedral faces. Note that $\Delta_{2}([0,1], 3)$ is not full and we need to pass to a barycentric subdivision. Right: the configuration space $\left|\operatorname{Sd}\left(X^{3}\right)\right|-\mid \operatorname{Sd}\left(\Delta_{2}(X, 3) \mid\right.$ deformation retracts onto the subcomplex $W^{2}([0,1], 3)$ made out of 6 contractible connected components. The figure shows one such component in one tetrahedral face.

Note that, as asserted, $\Delta_{2}([0,1], 3)$ is not full, as the 2 -simplex (bottom) $([0,0,0]$, $[1,0,0],[1,1,0])$ has all three vertices in $\Delta_{2}(X, 3)$ but is not itself a simplex of $\Delta_{2}(X, 3)$.

Generally a vertex is in $\Delta_{d+1}(X, n)$ if and only if it is of the form $\left(v_{1}, \ldots, v_{n}\right)$ for some vertices $v_{1}, \ldots, v_{n}$ of $X$ with $v_{i_{0}}=\cdots=v_{i_{d}}$ for some choice of sequence $i_{0}<i_{1}<\cdots<i_{d}$. Obviously every permutation acting on $X^{n}$ permutes vertices of $X_{*}^{n}$ and the order between them so it must take simplices to simplices. The action is simplicial and the quotient space $\operatorname{SP}^{n}(X)$ inherits a cellular decomposition. Moreover, the action remains simplicial after passing to a barycentric subdivision. Indeed since any new introduced vertex is of the form $\frac{1}{k} \sum v_{i}$, it is sent by $\sigma \in \mathfrak{S}_{n}$ to $\frac{1}{k} \sum \sigma\left(v_{i}\right)$, which is the barycenter of $\left(\sigma\left(v_{1}\right), \ldots, \sigma\left(v_{k}\right)\right)$.

After one subdivision, a simplicial neighborhood of $\operatorname{Sd}\left(\Delta_{d+1}(X, n)\right)$ consists of all simplices of $\operatorname{Sd}\left(X^{n}\right)$ having at least one vertex of the form $\left(v_{1}, \ldots, v_{n}\right)$ with $v_{i_{0}}=$ $\cdots=v_{i_{d}}$ for some sequence $i_{0}<i_{1}<\cdots<i_{d}$. This simplicial neighborhood is therefore $\mathfrak{S}_{n}$-invariant and its complement $W^{d}(X, n)$ is invariant, as well. Clearly the permutation action on $X^{n}$ commutes with Munkres' deformation since it takes combinations $\sum t_{i} v_{i}$ to $\sum t_{i} \sigma\left(v_{i}\right)$ (see Figure 2). It therefore descends to a deformation retraction of $B^{d}(X, n)$ onto $W^{d}(X, n) / \mathfrak{S}_{n}=: \mathcal{W}^{d}(X, n)$.

Corollary 5.3 For a finite simplicial complex $X$, the $\mathfrak{S}_{n}$-quotient $\mathcal{W}^{d}(X, n)$ of $W^{d}(X, n)$ is a compact deformation retract of $B^{d}(X, n)$.

We need one more observation.

Lemma 5.4 Let $A$ be a subcomplex of $X$. The deformation retraction of $\left|\operatorname{Sd}\left(X^{n}\right)\right|-$ $\left|\operatorname{Sd}\left(\Delta_{d+1}(X, n)\right)\right|$ onto its compactified space $W^{d}(X, n)$ restricts to a deformation retraction of $\left|\operatorname{Sd}\left(A^{n}\right)\right|-\left|\operatorname{Sd}\left(\Delta_{d+1}(A, n)\right)\right|$ onto $W^{d}(A, n)$. 

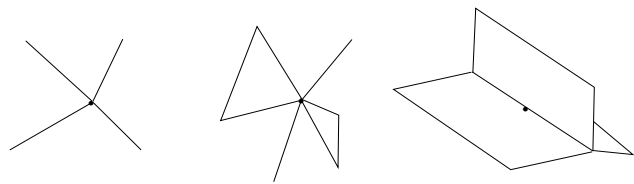

Figure 4: Contractible neighborhoods of the dotted point in simplicial $X$

Proof Since $A$ is a subcomplex of $X, \Delta_{d+1}(A, n)$ is a subcomplex of $\Delta_{d+1}(X, n)$ and $S d\left(A^{n}\right)$ is a subcomplex of $S d\left(X^{n}\right)$. Both $S d\left(\Delta_{d+1}(X, n)\right)$ and $S d\left(\Delta_{d+1}(A, n)\right)$ are full subcomplexes. The assertion now follows from the fact that if the deformation retraction starts in a simplex of $S d\left(X^{n}\right)$; in particular in $S d\left(A^{n}\right)$, it will stay in that simplex.

\section{Proof Theorem 1.1}

We appeal to the following useful theorem of Steve Smale which is a generalization of classical results of Begle and Vietoris. A similar statement for maps between simplicial complexes can be deduced from work of Farjoun [11, Corollary 9.B.3, page 163].

Theorem 6.1 [24] Let $X$ and $Y$ be connected, locally compact, separable metric spaces, and let $X$ be locally contractible. Let $f$ be a mapping of $X$ into $Y$ for which $f^{-1}$ carries compact sets into compact sets. If, for each $y \in Y, f^{-1}(y)$ is locally contractible and $r$-connected, $r \geq 0$, then the induced homomorphism $\pi_{k}(X) \rightarrow \pi_{k}(Y)$ is an isomorphism for $0 \leq k \leq r$, and is onto for $k=r+1$.

Theorem 6.1 uses maps that are proper and preimages that are at least connected. Maps between configuration spaces obtained by projections are seldom proper. Combining the above theorem with Section 5 yields, however, the following main result.

Theorem 6.2 Let $X$ be a connected finite simplicial complex with at least two vertices, $d \geq 2, n \geq 2$. Then

$$
\pi_{i}\left(B^{d}(X, n)\right) \cong \pi_{i}\left(\mathrm{SP}^{n}(X)\right), \quad 0 \leq i \leq 2 d-2 .
$$

Proof The starting point is Lemma 4.6 where it suffices to show that $\pi_{i}\left(B^{d}(V, n)\right)=0$ for $i \leq 2 d-2$ for $V$ a small contractible neighborhood of a point in $X$. A neighborhood $V$ of $x \in X$ is one of three types; either (i) Euclidean space, (ii) halfspace or (iii) it is a union of such halfspaces along a shared boundary. See Figure 4.

We claim that in all cases, $B^{d}(V, n)$ is $2 d-2-$ connected. 
In the case that $x$ is an interior point of a simplex that is not a face of a larger simplex, it has a neighborhood $V \cong \mathbb{R}^{m}$ with $m \geq 1$. When $m=1, B^{d}\left(\mathbb{R}^{1}, n\right)$ is contractible. When $m \geq 2, B^{d}\left(\mathbb{R}^{m}, n\right)$ is $2 d-2$-connected according to Proposition 4.10.

If $x$ belongs to a boundary face, then $V$ is homeomorphic to halfspace $H$ (with boundary). This halfspace can be isotoped into its interior $\stackrel{\circ}{H}$ so we have a map $B^{d}(H, n) \rightarrow B^{d}(\stackrel{\circ}{H}, n)$ obtained from a deformation retraction (setting $\left.t=1\right)$. Since $B^{d}(\stackrel{\circ}{H}, n)$ is $2 d-2-$ connected, as seen earlier, it follows immediately that $B^{d}(H, n)$ has the same connectivity (at least).

In the third and final case, $x$ lies in the intersection of two or more simplices of $X$ as in Figure 4. Let $V$ be a contractible neighborhood made out of simplices which meet along a simplex $A$. Let $\Gamma$ be a simplex in $V$ of dimension $m$. Of course $A$ is in the boundary of $\Gamma$. Let

$$
B^{d}(\Gamma, A, n)=\coprod_{0 \leq k \leq n} B_{A}^{d}(\Gamma, k) / \sim,
$$

where $B_{A}^{0}(\Gamma, k)=*$ is a given point in $A$ and $B_{A}^{d}(\Gamma, k)=B^{d}(\Gamma, k) \cup \operatorname{SP}^{k}(A)$, ie the only points that can repeat more than $d$ times in $\Gamma$ are those that are in $A$. The equivalence relation $\sim$ is such that $x \sim *$ if $x \in A$ and $\left[x_{1}, \ldots, x_{i}, \ldots, x_{k}\right] \sim$ $\left[x_{1}, \ldots, \hat{x}_{i}, \ldots, x_{k}\right]$ if $x_{i} \in A$. Here, as customary, $\hat{x}_{i}$ means the $i^{\text {th }}$ entry is suppressed. We have a projection

$$
\lambda: B^{d}(V, n) \rightarrow B^{d}(\Gamma, A, n),
$$

which sends a tuple $\left[x_{1}, \ldots, x_{n}\right]$ to the new tuple obtained by replacing all $x_{i} \notin \Gamma$ by $*$. One can view $\lambda$ as a projection of $\left[x_{1}, \ldots, x_{n}\right]$ to the subtuple made up of those entries $x_{i} \in \Gamma$. This map is continuous by the very nature of the construction $B^{d}(\Gamma, A, n)$, ie any entry $x_{i}$ that exits or enters into $\Gamma$ must pass through $A$. The base space $B^{d}(\Gamma, A, n)$ is contractible since there is a deformation retraction of $\Gamma$ onto $A$ which extends to $B^{d}(\Gamma, A, n)$.

Next write an element in $B^{d}(\Gamma, A, n)$ as an equivalence class $\left[\left[x_{1}, \ldots, x_{k}\right]\right]$ with $x_{i} \in \Gamma-A$ and some $k \leq n$. The preimage $\lambda^{-1}\left[\left[x_{1}, \ldots, x_{k}\right]\right]$ consists of all possible unordered $n$-tuples containing $x_{1}, x_{2}, \ldots, x_{k}$ with remaining entries $y_{1}, \ldots, y_{n-k}$ such that $\left[y_{1}, \ldots, y_{n-k}\right] \in B^{d}((V-\Gamma) \cup A, n-k)$. This preimage is a copy of $B^{d}((V-\Gamma) \cup A, n-k)$. By induction on the number of simplices of $V$, we can assume that $B^{d}((V-\Gamma) \cup A, n-k)$ is $2 d-2$-connected (the case of a single simplex has been discussed at the beginning of the proof). The map $\lambda: B^{d}(V, n) \rightarrow B^{d}(\Gamma, A, n)$ has then a contractible base and preimages that are $2 d-2$-connected. We wish to show that the total space is $2 d-2-$ connected. We cannot use the Smale-Vietoris theorem (Theorem 6.1) directly since $\lambda$ is not proper. To get around this, we pass 
to the compactified versions and show that $\lambda$ can be deformed to a proper map. Let $\mathcal{W}^{d}(V, n)$ be the compact deformation retract of $B^{d}(X, n)$ discussed in Corollary 5.3. The restriction $\tilde{\lambda}$ of $\lambda$ to $\mathcal{W}^{d}(V, n)$ maps onto $\mathcal{W}^{d}(\Gamma, A, n) \subset B^{d}(\Gamma, A, n)$ and we have the diagram

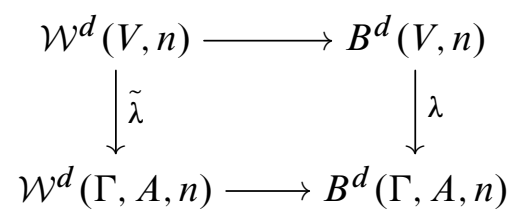

where the horizontal maps are inclusions and deformation retractions. This last statement follows from the fact that the deformation retraction of $B^{d}(V, n)$ onto $\mathcal{W}^{d}(V, n)$ descends to a deformation retraction of $B^{d}(\Gamma, A, n)$ onto $\mathcal{W}^{d}(\Gamma, A, n)$ as a consequence of Lemma 5.4. Thus given a configuration $\zeta=\left[\left[x_{1}, \ldots, x_{k}\right]\right] \in \mathcal{W}^{d}(\Gamma, A, n)$, $k \leq n, x_{i} \notin A$, we can consider its preimage $\tilde{\lambda}^{-1}(\zeta)$ in $\mathcal{W}^{d}(V, n)$ and its preimage $\lambda^{-1}(\zeta)$ in $B^{d}(V, n)$. Then $\lambda^{-1}(\zeta)$ deformation retracts onto $\tilde{\lambda}^{-1}(\zeta)$. Here $\tilde{\lambda}^{-1}(\zeta) \subset \lambda^{-1}(\zeta)=B^{d}((V-\Gamma) \cup A, n-k)$. Since $\lambda^{-1}(\zeta)$ is $2 d-2-$ connected, this shows that $\tilde{\lambda}^{-1}(\zeta)$ is also $2 d-2-$ connected. The map $\tilde{\lambda}$ is now proper, being a map between compact spaces. Moreover, both total and base spaces are connected by Lemma 4.1. We can invoke Theorem 6.1 to conclude that the total space $\mathcal{W}^{d}(V, n)$ and hence $B^{d}(V, n)$ are $2 d-2-$ connected as desired.

\section{Proof of Theorem 1.2}

Our objective is to find conditions on $X$ so that the inclusion $\Delta^{d}(X, n) \hookrightarrow X^{n}$ induces an isomorphism on some homotopy groups through a range (the homotopical depth). The proof given in the unordered case $B^{d}(X, n)$ in Section 6 fails here because the analogue of (7) is now a map $\Delta^{d}(V, n) \rightarrow \Delta^{d}(\Gamma, A, n)$ which has disconnected fibers, so Smale's theorem doesn't automatically apply. In fact we need an entirely new approach.

First some definitions.

Definition 7.1 - If $x \in X$ and $U$ is a neighborhood of $x$, then we call $V$ a subneighborhood (of $x$ in $U$ ) if $V$ is open and $x \in V \subset U$.

- A space $X$ is locally contractible if for any $x \in X$ and any neighborhood $U$ of $x$, there is a subneighborhood $V$ which deformation retracts onto $x$.

- A space $X$ has local homotopical dimension $k$ if, for $x, U$ as above, there is a subneighborhood $V$ such that $V-\{x\}$ is $k$-connected. For instance, being locally punctured connected means having 0 local homotopical dimension. A manifold of dimension $m$ has local homotopical dimension $m-2$ but not $m-1$. 
If $X$ is a simplicial complex, we call a chamber of $X$ any simplex that is not contained in another simplex as a face. Obviously if $X$ has local homotopical dimension $r$, then chambers must have dimensions at least $r+2$. We call a simplex a shared face if it is shared by two chambers or more. This shared face doesn't need to be of codimension 1 . In Figure 4, the complex on the far right is made out of three chambers (of dimension 2) joining along a shared edge. A shared face $A=\Gamma_{1} \cap \cdots \cap \Gamma_{k}$ is called essential if $X=\Gamma_{1} \cup \cdots \cup \Gamma_{k}$ is not a cell, ie homeomorphic to a ball or to a halfball. This rules out cases like $X$ being a regular polygon triangulated so that the origin $A=o$ is the common vertex of all triangles. A neighborhood $V \backslash\{o\}$ is, up to homotopy, a circle in this case, so that $o$ behaves like an interior point of a chamber (and is inessential).

Lemma 7.2 A finite simplicial complex $X$ has local homotopical dimension $r$ if and only if all chambers are of dimension at least $r+2$ and all essential shared faces are of dimension at least $r+1$.

Proof It suffices to consider points $x \in X$ that are either in the interior of a chamber or in the interior of a shared face. In the case that $x$ is in the interior of a chamber, $V \cong \mathbb{R}^{m}$ so $m$ (the dimension of the chamber) must be at least $r+2$ (Proposition 4.10). On the other hand, if $x$ lies in the interior of a shared face $A$, a small neighborhood $V$ of $x$ is the union of chambers $\Gamma_{1} \cup \cdots \cup \Gamma_{q}$ joining along $A$, with $q \geq 2$. If $A$ is inessential, then a neighborhood $V$ of $x \in A$ is either a ball or a halfball of dimension at least $r+2$. Suppose $x$ to be essential and let $s=\operatorname{dim} A$. Then $V-\{x\} \simeq \bigvee S^{s}$ is a bouquet (this holds even if $s=0$ and $A$ is vertex). Since this neighborhood must be $r$-connected, $s$ must be at least $r+1$.

The following is our main statement. Here we assume $d<n$; otherwise $\Delta^{d}(X, n)=X^{n}$ and there is nothing to prove.

Theorem 7.3 Let $X$ be a locally finite polyhedral space with local homotopical dimension $r, r \geq 0$, and let $1 \leq d<n$. Then

$$
\pi_{i}\left(\Delta^{d}(X, n)\right) \cong \pi_{i}(X)^{n} \text { for } i \leq r d+2 d-2
$$

Proof The starting point is Lemma 4.6. As in the proof of Lemma 7.2, a contractible neighborhood $V$ of $x \in X$ is one of three types: (i) $V \cong \mathbb{R}^{m}$ with $m \geq r+2$, (ii) halfspace $H$ of dimension $m \geq r+2$ or (iii) it is a union of such halfspaces along a shared face of dimension at least $r+1$. We must show that $\Delta^{d}(V, n)$ is $d r+2 d-2$ connected.

In the case $V \cong \mathbb{R}^{m}$, we know by Corollary 4.7 that $\Delta^{d}\left(\mathbb{R}^{m}, n\right)$ is $d m-2-$ connected, and that $d m-2=d(r+2)-2=d r+2 d-2$ as claimed. If $V$ is homeomorphic to 
halfspace $H$ (with boundary $L$ ), $V$ is the inverse limit of a nested sequence of spaces $H_{i} \supset H$ such that $H_{i} \cong \mathbb{R}^{m}$ for all $i$, it follows that $\Delta^{d}(H, n)$ is the inverse limit of the spaces $\Delta^{d}\left(H_{i}, n\right)$, which are $d r+2 d-2-$ connected, and it has this same connectivity. We are left with the case that $V$ is the union of simplices (chambers) $\Gamma_{1} \cup \cdots \cup \Gamma_{q}$ joining along an essential face $A$. We can assume without loss of generality that any two faces join along $A$, ie $\Gamma_{i} \cap \Gamma_{j}=A$. Luckily the structure of this neighborhood $V$ is sufficiently nice to allow us to give a decomposition of $\Delta^{d}(V, n)$ as the colimit of an explicit diagram.

We start by observing that each configuration of $n$ points of $V$ gives rise to a tuple of integers $\left(k_{1}, \ldots, k_{q}\right), k_{1}+\cdots+k_{q} \geq n$, where $k_{i}$ denotes the number of points of the configuration inside the face $\Gamma_{i}$. Obviously these $k_{i}$-configurations can overlap when points of the configuration fall in $A$. Keeping track of the various overlaps can be expressed in terms of a poset of intersections. More precisely, set the index set

$$
I=\{1,2, \ldots, q\}^{n}=\left\{\left(i_{1}, \ldots, i_{n}\right) \mid i_{j} \in\{1,2, \ldots, q\}\right\} .
$$

We can cover $\Delta^{d}(V, n)$ by the closed sets $U_{\left(i_{1}, \ldots, i_{q}\right)},\left(i_{1}, \ldots, i_{q}\right) \in I$, where

$$
U_{\left(i_{1}, \ldots, i_{q}\right)}=\left\{\left(x_{1}, \ldots, x_{n}\right) \in \Delta^{d}(V, n) \mid x_{j} \in \Gamma_{i_{j}}, i_{j} \in\{1,2, \ldots, q\}\right\} .
$$

Let $\mathcal{D}$ be the intersection poset $P_{U}$ associated to the cover $U_{I}$ of $\Delta^{d}(V, n)$, also referred to as subspace diagram. It is clear by construction that $\operatorname{colim} \mathcal{D}$ is precisely $\Delta^{d}(V, n)$. Here's how this poset diagram looks for $k=2$ and $d=1$, ie for the configuration space $F\left(X \cup_{A} Y, 2\right)$; see [25, Theorem 2.0.17]:
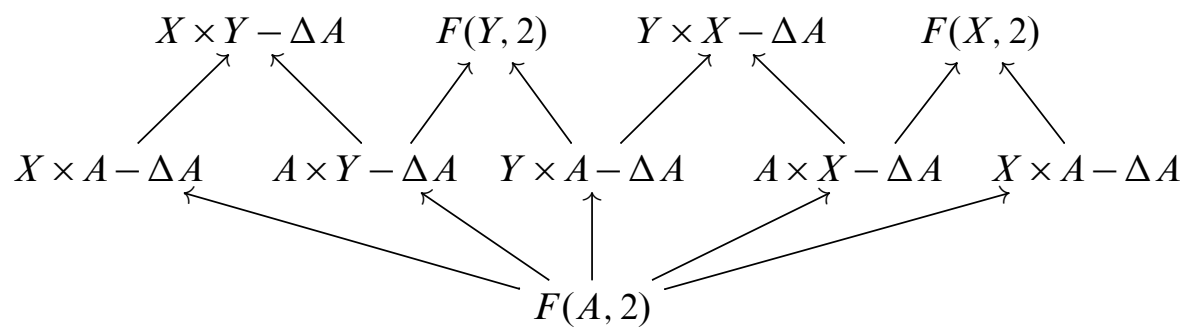

(the spaces on the extreme right and left are being identified).

Going back to the general diagram $\mathcal{D}$, since all inclusions are closed cofibrations (this is standard to check [25]), we have

$$
\Delta^{d}(V, n)=\operatorname{colim}(\mathcal{D}) \simeq \operatorname{hocolim}(\mathcal{D}) .
$$

In fact the canonical map from the homotopy colimit of a sequence of inclusions of $\mathrm{T} 1$ topological spaces to the actual colimit is a weak equivalence; see [7]. The connectivity of this (sequential) homotopy colimit $\Delta^{d}(V, n)$ is at least the least connectivity of 
the spaces making up the diagram. If we set $\Gamma_{0}=A$, these spaces are of the form $\Gamma_{i_{1}} \times \cdots \times \Gamma_{i_{n}} \cap \Delta^{d}(V, n)$ (we refer to these subspaces as the constituent subspaces of the diagram). Each of these constituent subspaces is quite manageable and we can apply the localization principle to it. Indeed $\Gamma_{i_{1}} \times \cdots \times \Gamma_{i_{n}} \cap \Delta^{d}(V, n)$ is the complement in $\Gamma_{i_{1}} \times \cdots \times \Gamma_{i_{n}}$ of subspaces of certain codimensions. The smallest codimension is attained by $\Delta_{d+1}(A, n)$ in $A^{n}$, that is, for $\Delta^{d}(A, n)$. If $s=\operatorname{dim} A$, then this codimension is $d s$. It follows that the smallest connectivity among the constituent subspaces is $d s-2 \geq d(r+1)-2=d r+d-2$. As pointed out, the connectivity of $\Delta^{d}(V, n)$ (as a homotopy colimit) must be at least the connectivity of $\Delta^{d}(A, n)$, which is $d r+d-2$. This is not quite the connectivity we seek and we must improve it by $d$.

To do so observe that there is associated to the poset $P_{U}$ of the cover a natural filtration whose $j^{\text {th }}$ space is $\mathcal{F}_{j}=\operatorname{colim} P_{j}$, where $P_{j}$ is the poset consisting of

$$
\begin{gathered}
\Gamma_{i_{1}} \times \cdots \times \Gamma_{i_{n}} \cap \Delta^{d}(V, n), \quad i_{k_{1}}=\cdots=i_{k_{s}}=0 \quad \text { for } s \geq n-j, \\
\quad \text { and some subset }\left\{k_{1}, \ldots, k_{s}\right\} \subset\{1, \ldots, n\}
\end{gathered}
$$

with $i_{s} \in\{0,1, \ldots, q\}$ and $\Gamma_{0}=A$, as pointed out. In other words, $\mathcal{F}_{j}$ is the subspace where at most $j$ of the entries can be outside of $A$. We have the series of inclusions

$$
\mathcal{F}_{0}=\Delta^{d}(A, n) \subset \mathcal{F}_{1} \subset \cdots \subset \mathcal{F}_{n}=\Delta^{d}(V, n) .
$$

If we organize our poset vertically as in (8), then $\mathcal{F}_{j}$ is the pushout of the first $j+1$ rows from the bottom.

For example, $F\left(\Gamma_{1} \cup_{A} \Gamma_{2}, 2\right)$ (the case depicted in diagram (8) with $\left.X=\Gamma_{1}, Y=\Gamma_{2}\right)$, there are three filtration terms starting with $\mathcal{F}_{0}=F(A, 2)$, the colimit $\mathcal{F}_{1}$ of the first two rows and $\mathcal{F}_{2}$ being the whole colimit. The special case of $F\left(\mathbb{R}^{2}, 2\right)=\Delta^{1}\left(\mathbb{R}^{2}, 2\right)$ is enlightening $(d=1, n=2)$, where here we write $\mathbb{R}^{2}=\Gamma_{1} \cup_{A} \Gamma_{2}$ with the $\Gamma_{i}$ being two halfplanes joining along $A \cong \mathbb{R}$. The first filtration term is $\mathcal{F}_{0}=F(\mathbb{R}, 2) \simeq S^{0}$. The next filtration term is

$$
\mathcal{F}_{1}=\left(\Gamma_{1} \times A \cup A \times \Gamma_{1} \cup \Gamma_{2} \times A \cup A \times \Gamma_{2}\right) \cap \Delta^{1}\left(\mathbb{R}^{2}, 2\right) .
$$

Each term $\left(\Gamma_{i} \times A \cup A \times \Gamma_{j}\right) \cap \Delta^{1}\left(\mathbb{R}^{2}, 2\right)=\Gamma_{i} \times A \cup A \times \Gamma_{i}-\operatorname{diag}(A)$ deformation retracts onto a circle so $\mathcal{F}_{1}$ is the union of two circles along an $S^{0}$, ie $\mathcal{F}_{1} \simeq S^{1} \vee S^{1} \vee S^{1}$. Finally $\mathcal{F}_{2} \simeq F\left(\mathbb{R}^{2}, 2\right) \simeq S^{1}$. The connectivity changes going from $\mathcal{F}_{0}$ to $\mathcal{F}_{1}$, and remains stable afterwards.

Let's organize into a row $R_{k}$ the constituent subspaces $\Gamma_{i_{1}} \times \cdots \times \Gamma_{i_{n}} \cap \Delta^{d}(V, n)$ where precisely $k$ of the $\Gamma_{i_{j}}$ are not equal to $A=\Gamma_{0}$. One point we will capitalize on is that in the range $0 \leq k \leq d, \Gamma_{i_{1}} \times \cdots \times \Gamma_{i_{n}} \cap \Delta^{d}(V, n)$ is the complement in 
$\Gamma_{i_{1}} \times \cdots \times \Gamma_{i_{n}}$ of tuples with $d+1$-diagonal elements lying only in $A$. At the first stage, all components of $R_{1}$ intersect along $\mathcal{F}_{0}=\Delta^{d}(A, n)$.

If $n=d+1$, the situation is very clear. Here $\Delta^{d}(A, d+1)=A^{d+1}-\operatorname{diag}(A) \simeq S^{d s-1}$, and all constituent subspaces for $1 \leq k \leq d$ are of the form

$$
\Gamma_{i_{1}} \times \cdots \times \Gamma_{i_{d+1}} \cap \Delta^{d}(V, d+1)=\Gamma_{i_{1}} \times \cdots \times \Gamma_{i_{d+1}}-\operatorname{diag}(A),
$$

thus they are contractible since they are the complement of a closed subspace in the boundary of a cube. This means that going up the filtration, we are suspending in various ways the spherical class, as in the example discussed earlier, and the connectivity in homology is going up by one at every step.

For more general $n$, the constituent subspaces are not, in general, contractible but we have the following useful lemma.

Lemma 7.4 The inclusion $\mathcal{F}_{k-1} \hookrightarrow \mathcal{F}_{k}$ is null-homotopic for $k \leq d+1$.

Proof We need some notation. We introduce $\mathcal{F}_{k}(n)$ for the filtration terms of $\Delta^{d}(V, n)$ (we added the index $n$ to the previous notation). We also introduce $\mathcal{F}_{k, j}(n)$ for the subspace of all configurations $\left(x_{1}, \ldots, x_{n}\right) \in \mathcal{F}_{k}(n)$ where $x_{j}$ can be in all of $H$. We have that $\mathcal{F}_{k}(n)=\bigcup_{1 \leq j \leq n} \mathcal{F}_{k, j}(n)$. There is an inclusion

$$
\mathcal{F}_{k-1}(n) \hookrightarrow \mathcal{F}_{k, n}(n) \subset \mathcal{F}_{k}(n) .
$$

On the other hand there are various embeddings of $\mathcal{F}_{j}(n)$ into $\mathcal{F}_{j}(n+1)$ one of which is given by

$$
\left(x_{1}, \ldots, x_{n}\right) \mapsto\left(\phi_{1}\left(x_{1}\right), \ldots, \phi_{1}\left(x_{n}\right), p_{n}\right),
$$

where $\phi_{t}$ is any isotopy of the halfspace $H$ extending an isotopy of $A$ onto its halfspace $\left(a_{1}, \ldots, a_{s}\right), a_{1}<0$, and $p_{n}=(n, 0, \ldots, 0) \in A \cong \mathbb{R}^{s}$. The first observation is that the inclusion $\mathcal{F}_{k-1}(n) \hookrightarrow \mathcal{F}_{k, n}(n)$ is homotopic to the composite

$$
\mathcal{F}_{k-1}(n) \rightarrow \mathcal{F}_{k-1}(n-1) \hookrightarrow \mathcal{F}_{k-1}(n) \hookrightarrow \mathcal{F}_{k, n}(n),
$$

where the first map is projection discarding the last configuration, and the middle map is the inclusion (9). The idea here is that the last coordinate $x_{n} \in H$ can be moved in $H$ away from $A$, all configurations are then mapped by $\phi_{t}$, and after that the last coordinate is brought down to $p_{n}$. Note that the last configuration can move in $H$ without constraint since $k \leq d$. Next we factor the composite above $\mathcal{F}_{k-1}(n-1) \hookrightarrow \mathcal{F}_{k}(n)$ through $\mathcal{F}_{k-1}(n-1) \hookrightarrow \mathcal{F}_{k, n-1}(n)$ and reiterate this construction to factor the map up to homotopy, this time through $\mathcal{F}_{k-1}(n-2)$, etc. At the end, the map $\mathcal{F}_{k-1}(n) \hookrightarrow \mathcal{F}_{k}(n)$ factors through $\mathcal{F}_{k-1}(k-1)$ which is contractible. 
Going back to our colimit diagram, the constituent subspaces for the $k^{\text {th }}$ row $R_{k}$ are $V_{I}:=\Gamma_{i_{1}} \times \cdots \times \Gamma_{i_{n}} \cap \Delta^{d}(V, n)$, where $I=\left(i_{1}, i_{2}, \ldots, i_{n}\right)$ is an ordered tuple with $n-k$ entry 0 . In the range $k<n-d$, the smallest connectivity of a constituent subspace is $d s-2$; this is because the smallest codimension strata we are removing from $\Gamma_{i_{1}} \times \cdots \times \Gamma_{i_{n}}$ to obtain $V_{I}$ have the codimension of the thin diagonal in $A^{d+1}$ and this is $d s$. In the range $n-d \leq k<n$, this minimal codimension starts jumping by one unit going from row to row. More precisely the connectivity of the constituent subspaces of $R_{k}$ in the indicated range is at least $d s-2+k-(n-d-1)$. This minimal connectivity remains the same from $R_{n-1}$ to $R_{n}$ (no jump there). There are therefore precisely $d$ jumps. At the level of filtrations now, $\mathcal{F}_{k}=R_{k} \cup \mathcal{F}_{k-1}$ and we have a pushout diagram where we are gluing $d s-2+k-(n-d-1)-$ connected spaces intersecting along $d s-2+k-(n-d-1)-1-$ connected spaces. Using the MayerVietoris sequence, and inducting on the sequences $I$, we see immediately that the homological connectivity (in short $H_{*}$-connectivity) of the pushout $\mathcal{F}_{k}$ must be at least $d s-2+k-(n-d-1)$, for $n-d \leq k<n$. The $H_{*}$-connectivity of $\mathcal{F}_{n}$ is, as we pointed out, that of $\mathcal{F}_{n-1}$, which is thus at least $d s-2+d$. Since $s \geq r+1$ (Lemma 7.2), this $H_{*}$-connectivity is at least $d r+2 d-2-$ connected.

Finally to get the connectivity, we need argue that $\mathcal{F}_{n}$ is simply connected. In fact $\mathcal{F}_{k}$ becomes 1 -connected as soon as $k \geq 1$. To see this, we go back to the colimit diagram (8) where the smallest connectivity of the constituent subspaces $V_{I}$ is $d(r+1)-2$. When this is larger than 1 , each $V_{I}$ is simply connected, and so is the colimit, and the theorem holds. Now some $V_{I}$ fail to be simply connected when $d(r+1) \leq 2$, that is, when (i) $r=0, d=1$, or (ii) $r=1=d$, or (iii) $r=0, d=2$. In the first case, the theorem is equivalent to saying that $F(X, n)$ is connected if $X$ is locally punctured connected. This is precisely Lemma 4.1 so this case is settled. In case (ii), we are looking at $\Delta^{d}(A, n)=\Delta^{1}\left(\mathbb{R}^{2}, n\right)=F\left(\mathbb{R}^{2}, n\right)$ as the bottom space of our colimit diagram. This is of course not simply connected, but the map $\pi_{1}\left(\mathcal{F}_{0}\right) \rightarrow \pi_{1}\left(\mathcal{F}_{1}\right)$ is the trivial map since it is induced from a null homotopic map (Lemma 7.4), so that $\mathcal{F}_{1}$, and inductively $\mathcal{F}_{k}$, are simply connected by the van Kampen theorem. The remaining case (iii) occurs when $\Delta^{d}(A, n)=\Delta^{2}(\mathbb{R}, n)$. The fundamental group of this space is discussed in Example 8.1. Here too the fundamental group trivializes from $\mathcal{F}_{1}$ onwards so that $\mathcal{F}_{n}=\Delta^{d}(V, n)$ is simply connected.

\section{A The homology of the filtration terms}

This subsection is of independent interest and gives a description of the homology of the filtration terms. This is sketchy but details can be filled in. First of all, there is a nice way to see that the inclusion $\mathcal{F}_{0} \rightarrow \mathcal{F}_{1}$ induces the trivial map in homology without 
resorting to Lemma 7.4. Here $\mathcal{F}_{0}=\Delta^{d}(A, n)$ has torsion free homology admitting a basis realized by products of spheres [9]. We need to understand how these homology classes occur. There is a spherical class in

$$
\Delta^{d}(A, d+1)=A^{d+1}-\operatorname{diag}(A) \simeq S^{d s-1},
$$

where $s=\operatorname{dim} A$. Now $\Delta^{d}(A, d+1)$ embeds in $\Delta^{d}(A, n)$ in many ways as in (9) (recall that $d<n)$. This embedding has a retract so induces a monomorphism in homology. The image of the spherical class in this case is denoted $\left\{x_{1}, \ldots, x_{d+1}\right\}$. The various other embeddings, obtained by choosing another subset of indices $\left\{i_{1}, \ldots, i_{d+1}\right\} \subset$ $\{1,2, \ldots, n\}$, give rise to spherical homology classes $\left\{x_{i_{1}}, \ldots, x_{i_{d+1}}\right\}$. These classes generate the homology of $\Delta^{d}(A, n)$ in a very precise sense. There is an action of the operad $\left\{D^{s}(k)\right\}_{k \geq 0}$ of little $s$-dimensional disks on $\bigcup_{n \geq 1} \Delta^{d}(A, n)$, where $s=\operatorname{dim} A$ and $D^{s}(k)$ is the space of $k$ pairwise disjoint open disks in the unit disk of dimension $s$ (to keep with the terminology the word "disk" is used instead of "ball"). The action of $D^{s}(2) \simeq S^{s-1}$ is given as follows:

$$
D^{s}(2) \times \Delta^{d}\left(A, n_{1}\right) \times \Delta^{d}\left(A, n_{2}\right) \rightarrow \Delta^{d}\left(A, n_{1}+n_{2}\right),
$$

and yields a bracket operation in homology:

$$
[-,-]: H_{p}\left(\Delta^{d}\left(A, n_{1}\right)\right) \otimes H_{q}\left(\Delta^{d}\left(A, n_{2}\right)\right) \rightarrow H_{p+q+s-1}\left(\Delta^{d}\left(A, n_{1}+n_{2}\right)\right) .
$$

The product map in homology is given by the action of $H_{0}\left(D^{s}(2)\right)$ and is the induced map in homology of the concatenation of two configurations after placing the first one in a disk of radius $\frac{1}{2}$ centered at $\left(-\frac{1}{2}, 0, \ldots, 0\right)$ and the other in another disk of the same radius centered at $\left(\frac{1}{2}, 0, \ldots, 0\right)$. One main theorem of [9] reads as follows. The bracket of two cycles is important to understand and can be described as follows. Given a cycle (or chain) $c$ in $\Delta^{d}(A, n)$, we say we localize it in a disk $D^{s}$ if we choose a homeomorphism (which can be made canonical) between $A \cong \mathbb{R}^{s}$ and $D$, and take the image of $c$ in $\Delta^{d}(D, n)$ via this homeomorphism. We obtain the bracket $\left[\alpha_{1}, \alpha_{2}\right]$ by localizing the cycles respectively in two disjoints disks $D_{1}$ and $D_{2}$ and taking the new cycle obtained by rotating $D_{1}$ around $D_{2}$ (or $D_{2}$ around $D_{1}$, up to sign) in $\mathbb{R}^{s}$.

Theorem 7.5 [9, Proposition 3.9] The homology of $\Delta^{d}(A, n)$ is torsion free, generated additively by products of iterated brackets where each factor is either $x_{i}$ or an iterated bracket of the form

$$
\left[\cdots\left[\left[B_{1}, B_{2}\right], B_{3}\right], \ldots, B_{\ell}\right], \quad \ell \geq 1,
$$

where each $B_{S}$ is of the form

$$
B_{s}=\left[\cdots\left[\left[\left\{x_{j_{1, s}}, x_{j_{2, s}}, \ldots, x_{j_{d+1, s}}\right\}, x_{i_{1, s}}\right], x_{i_{2, s}}\right], \ldots, x_{i_{\ell_{s}, s}}\right]
$$

(further conditions are stated on indices to get a basis). 
Let's argue, for example, that $\left\{x_{1}, \ldots, x_{d+1}\right\}$ maps to zero in the homology of the next filtration term. Consider the following diagram of inclusions:

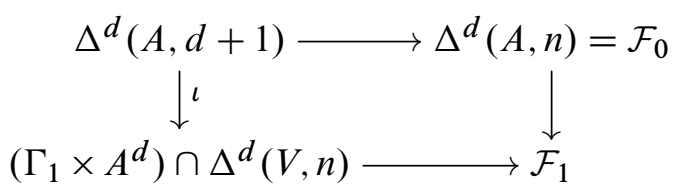

The bottom space $\left(\Gamma_{1} \times A^{d}\right) \cap \Delta^{d}(V, n)=\Gamma_{1} \times A^{d}-\operatorname{diag}(A)$ is contractible since we are removing a subspace from the boundary of $\Gamma_{1} \times A^{d}$. The map $\iota$ is trivial and the commutativity of the diagram shows that $\left\{x_{1}, \ldots, x_{d+1}\right\}$ maps trivially in $\mathcal{F}_{1}$. A class of the form $\left[\left\{x_{1}, \ldots, x_{d+1}\right\}, x_{d+2}\right]$ dies in $\mathcal{F}_{1}$, for example, since this class can be represented by the composite

$$
S^{d s-1} \times S^{d-1} \rightarrow A^{d+2}-\operatorname{sing} \hookrightarrow A^{d+1} \times H-\operatorname{sing} \hookrightarrow \mathcal{F}_{1} .
$$

The first map is obtained from the operadic action. Here the factor $S^{d-1}$ is the locus of $x_{d+2}$ rotating in some sphere in $\mathbb{R}^{d}$, so when $x_{d+2}$ is allowed to be in $H$, this sphere is coned off and the composite of the first two maps in (10) is trivial on the top homology class which by definition is $\left[\left\{x_{1}, \ldots, x_{d+1}\right\}, x_{d+2}\right]$. A similar argument applies to show that the image of $B_{s}$ as in the notation of Theorem 7.5 is trivial in $\mathcal{F}_{1}$. For the image of the bracket $\left[B_{S}, B_{t}\right]$, one can argue similarly. One constructs this class by localizing $B_{s}$ and $B_{t}$ in distinct disks $D_{1}$ and $D_{2}$, and rotating one disk around the other. But the class $B_{S}$ is the boundary of a chain in $H^{\prime} \times D_{1}^{n-1} \cup D_{1} \times H^{\prime} \times D^{n-2} \cup \cdots \cup D^{n-1} \times H^{\prime} \subset \mathcal{F}_{1}$, where $H^{\prime}$ is the part of $H$ with boundary $D_{1}$. This means that $\left[B_{s}, B_{t}\right]$ must map to zero in $H_{*}\left(\mathcal{F}_{1}\right)$. It remains to be shown that the image of a product is trivial, but this is immediate.

Note that there are many ways a given class $\left[\cdots\left[\left[B_{1}, B_{2}\right], B_{3}\right], \ldots, B_{\ell}\right]$ can die in $\mathcal{F}_{1}$, and so in $\mathcal{F}_{1}$ we obtain suspension classes one degree higher. This describes the homology of $\mathcal{F}_{1}$ and clearly it is one degree more connected than $\mathcal{F}_{0}$.

\section{Fundamental groups}

In this final section we take a more pedestrian look at the isomorphism $\pi_{1}\left(B^{d}(X, n)\right) \cong$ $H_{1}(X, \mathbb{Z})$ for $d \geq 2$. This is expressed in terms of braids. As before $X$ is a simplicial complex. Note that loops in $\mathrm{SP}^{n} X$, based at a basepoint of the form $[*, \ldots, *]$, say, lift to $X^{n}$ under the quotient projection; see [18, Section 5] for example:

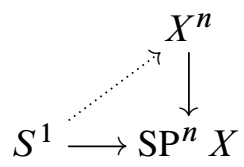



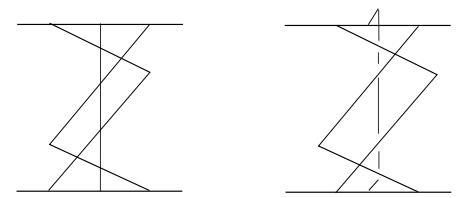

Figure 5: The left braid cannot be trivialized in $\Delta^{2}(I, 3)$

This says that a homotopy class of a loop $\gamma: S^{1} \rightarrow \mathrm{SP}^{n} X$ based at $[*, \ldots, *]$ can be represented by a tuple $\left[\gamma_{1}, \ldots, \gamma_{n}\right]$, where $\gamma_{i}: S^{1} \rightarrow X$ is a loop in $X$. Moreover and by the simplicial approximation theorem, any loop in $X^{n}$ deforms into an $n$-tuple of simplicial loops in $X$ so that $\gamma$ can be represented by an unordered tuple of simplicial loops in $\mathrm{SP}^{n}(X)$ for some simplicial decomposition.

We can try to describe loops in $\Delta^{d}(X, n)$ and $B^{d}(X, n)$ in the same way but both spaces are not simplicial complexes in general, only of the homotopy type of one. However, after passing to a barycentric subdivision, $B^{d}(X, n)=\operatorname{SP}^{n} X-B_{d+1}(X, n)$ deformation retracts onto a cellular complex $\mathcal{W}^{d}(X, n)$ (Lemma 5.2). A loop $S^{1} \rightarrow$ $B^{d}(X, n)$ deforms into a loop into $\mathcal{W}^{d}(X, n)$ which is cellular. Therefore and without loss of generality, we can represent a loop $\gamma: S^{1} \rightarrow B^{d}(X, n)$ within its homotopy class by a tuple of paths $t \mapsto\left[\gamma_{1}(t), \ldots, \gamma_{n}(t)\right]$, with $\gamma_{i}$ a simplicial path in $X$ (not necessarily a closed loop) and $t \in[0,1]$. This is a braid with $n$-strands. These paths or strands at any time $t$ do not intersect in more than $d$ points, and $\left[\gamma_{1}(0), \ldots, \gamma_{n}(0)\right]=$ $\left[\gamma_{1}(1), \ldots, \gamma_{n}(1)\right]$. This is similar for loops into $\Delta^{d}(X, n)$.

As a first example, consider $X=I$ : the unit interval. By codimension argument, $\Delta^{d}(I, n)$ is simply connected if $d \geq 3$, so the only interesting case is when $d=2$ and we are removing from $I^{n}$ codimension 2 subspaces corresponding to when $x_{i}=x_{j}=x_{k}$. According to Example 2.1, $\Delta^{2}(I, 3) \simeq S^{1}$ and $\pi_{1}\left(\Delta^{2}(I, 3)\right) \cong \mathbb{Z}$. An element in the fundamental group can be represented by a braid with 3 -strands embedded in $I \times I$, not all of which can pass by the same point at the same time. A nontrivial element is depicted in the left-hand side of Figure 5. This braid cannot be trivialized in $\Delta^{2}(I, 3)$, but it is amusing to try. By moving the strands around while keeping their endpoints fixed, there is no way we can separate them without going through a triple point.

Example 8.1 For $n \geq 3$, the fundamental group of $\Delta^{2}(I, n)$ has been analyzed by Khovanov [19]. There he shows that $\Delta^{2}(I, n)$ is a $K(\pi, 1)$ and then gives a presentation for $\pi$. This presentation is given as follows. Define the right-angled Coxeter group $T W_{n}$ to be the group generated by the simple transpositions $s_{i}=(i, i+1), i \in[n-1]$, subject to the relations

$$
s_{i}^{2}=1, \quad s_{i} s_{j}=s_{j} s_{i} \text { if }|i-j|>1 .
$$

Define $\phi: T W_{n} \rightarrow \mathfrak{S}_{n}$ by $\phi\left(s_{i}\right)=s_{i}$ for all $i \in[n-1]$. Then $\pi_{1}\left(\Delta^{2}(I, n)\right) \cong \operatorname{ker} \phi$. 


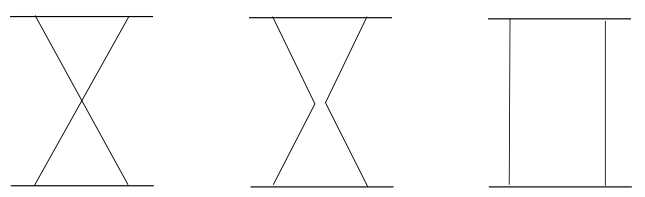

Figure 6: Resolving the intersection points

In the unordered case it is possible to kill the braiding by interchanging strands. Represent an element of $\pi_{1}\left(B^{d}(X, n)\right)$ by a braid with $n$-strands embedded in $X \times I$. Suppose we have two intersecting strands. There is a way to resolve the intersection points, illustrated in Figure 6. The figure depicts a loop $f(t)=\left[f_{1}(t), f_{2}(t)\right]$ with two strands crossing for some $s \in[0,1]$. Define $\tilde{f}=\left[\tilde{f}_{1}, \tilde{f}_{2}\right]$ to be such that $\tilde{f}_{i}(t)=f_{i}(t)$ if $t \leq s$, and $\tilde{f}_{1}(t)=f_{2}(t), \tilde{f}_{2}(t)=f_{1}(t)$ if $t \geq s$. These give two representations of the same loop in $\Omega B^{d}(X, n)$ for $d \geq 2$. The difference, however, is that after changing $f$ by $\tilde{f}$, by a small homotopy we can now separate the strands of $\tilde{f}$ so that no intersection occurs. This also explains why the fundamental group must be abelian [18].

For example, using this resolution of intersections, we can immediately trivialize the braid in $\Omega B^{2}(\mathbb{R}, 3)$ depicted in Figure 5 (left). This is no surprise since $B^{2}(\mathbb{R}, 3) \simeq$ $B^{2}(I, 3)$ is contractible and is identified with the 3 -simplex with one edge removed.

The resolution of intersections when applied to loops in $\Omega V$, with $V$ a tree, implies that we have a surjection $\pi_{1}(B(V, n)) \rightarrow \pi_{1}\left(B^{d}(V, n)\right)$. Since $B^{d}(V, n)$ is connected for $d \geq 2$ (Lemma 4.1), pick the basepoint in this fundamental group to be $\left[x_{1}, \ldots, x_{n}\right]$ with $x_{i} \neq x_{j}, i \neq j$, and write a braid $\gamma(t)=\left[\gamma_{1}(t), \ldots, \gamma_{n}(t)\right]$. As discussed, we can assume the $\gamma_{i}$ to be nonintersecting strands. Since $V$ is one dimensional, necessarily $\gamma_{i}(0)=\gamma_{i}(1)=x_{i}$, so all strands must start and finish at the same point. Each $\gamma_{i}$ can be homotoped to the constant strand at $x_{i}$, without further intersections, and the loop we started out with is trivial up to homotopy. The above discussion allows us to give a streamlined proof of the following proposition which we have already obtained as a corollary to Theorem 1.1.

Proposition 8.2 If $X$ is a connected simplicial complex which is not reduced to a point, $n \geq 2, d \geq 2$, then there is an isomorphism $\pi_{1}\left(B^{d}(X, n)\right) \cong H_{1}(X ; \mathbb{Z})$.

Proof We need show that the inclusion $B^{d}(X, n) \hookrightarrow \mathrm{SP}^{n} X$ induces an isomorphism on fundamental group if $d>1$. If we invoke Lemma 4.6 as before, this boils down to showing that for $V$ a contractible neighborhood in $X, B^{d}(V, n)$ is simply connected whenever $d \geq 2$ and for any $n \geq 1$. If $V$ is a contractible neighborhood in simplicial $X$ (as in the proof of Theorem 6.2), any element in $\pi_{1}\left(B^{d}(V, n)\right.$ ) can be represented by a braid and by resolving the intersection points. This braid can be homotoped to the trivial braid. 


\section{References}

[1] M A Armstrong, The fundamental group of the orbit space of a discontinuous group, Proc. Cambridge Philos. Soc. 64 (1968) 299-301 MR0221488

[2] A Björner, M Las Vergnas, B Sturmfels, N White, G M Ziegler, Oriented matroids, 2nd edition, Encyclopedia of Mathematics and its Applications 46, Cambridge Univ. Press (1999) MR1744046

[3] A Björner, V Welker, The homology of " $k$-equal" manifolds and related partition lattices, Adv. Math. 110 (1995) 277-313 MR1317619

[4] P V M Blagojević, B Matschke, G M Ziegler, A tight colored Tverberg theorem for maps to manifolds, Topology Appl. 158 (2011) 1445-1452 MR2812494

[5] P V M Blagojević, G M Ziegler, Convex equipartitions via equivariant obstruction theory, Israel J. Math. 200 (2014) 49-77 MR3219570

[6] C-F Bödigheimer, I Madsen, Homotopy quotients of mapping spaces and their stable splitting, Quart. J. Math. Oxford Ser. 39 (1988) 401-409 MR975905

[7] B Branman, I Kriz, A Pultr, A sequence of inclusions whose colimit is not a homotopy colimit, New York J. Math. 21 (2015) 333-338 MR3358546

[8] F Cohen, E L Lusk, Configuration-like spaces and the Borsuk-Ulam theorem, Proc. Amer. Math. Soc. 56 (1976) 313-317 MR0425949

[9] N Dobrinskaya, V Turchin, Homology of non-k-overlapping discs, Homology Homotopy Appl. 17 (2015) 261-290 MR3426383

[10] C Eyral, Profondeur homotopique et conjecture de Grothendieck, Ann. Sci. École Norm. Sup. 33 (2000) 823-836 MR1832992

[11] E D Farjoun, Cellular spaces, null spaces and homotopy localization, Lecture Notes in Mathematics 1622, Springer (1996) MR1392221

[12] MA Guest, A Kozlowski, K Yamaguchi, Stable splitting of the space of polynomials with roots of bounded multiplicity, J. Math. Kyoto Univ. 38 (1998) 351-366 MR1648299

[13] A Hatcher, Algebraic topology, Cambridge Univ. Press (2002) MR1867354

[14] U Helmke, Topology of the moduli space for reachable linear dynamical systems: the complex case, Math. Systems Theory 19 (1986) 155-187 MR871414

[15] T D Imbo, C Shah Imbo, E C G Sudarshan, Identical particles, exotic statistics and braid groups, Phys. Lett. B 234 (1990) 103-107 MR1036962

[16] S Kallel, Spaces of particles on manifolds and generalized Poincaré dualities, Q. J. Math. 52 (2001) 45-70 MR1820902

[17] S Kallel, R Karoui, Symmetric joins and weighted barycenters, Adv. Nonlinear Stud. 11 (2011) 117-143 MR2724545 
[18] S Kallel, W Taamallah, The geometry and fundamental group of permutation products and fat diagonals, Canad. J. Math. 65 (2013) 575-599 MR3043042

[19] M Khovanov, Real $K(\pi, 1)$ arrangements from finite root systems, Math. Res. Lett. 3 (1996) 261-274 MR1386845

[20] B Kloeckner, The space of closed subgroups of $\mathbb{R}^{n}$ is stratified and simply connected, J. Topol. 2 (2009) 570-588 MR2546586

[21] K H Ko, H W Park, Characteristics of graph braid groups, Discrete Comput. Geom. 48 (2012) 915-963 MR3000570

[22] J R Munkres, Elements of algebraic topology, Addison-Wesley Publishing Company, Menlo Park, CA (1984) MR755006

[23] M Nakaoka, Cohomology of symmetric products, J. Inst. Polytech. Osaka City Univ. Ser. A 8 (1957) 121-145 MR0102069

[24] S Smale, A Vietoris mapping theorem for homotopy, Proc. Amer. Math. Soc. 8 (1957) 604-610 MR0087106

[25] Q Sun, Configuration spaces of singular spaces, $\mathrm{PhD}$ thesis, University of Rochester, Ann Arbor, MI (2010) MR2890113 Available at http://search.proquest.com/ docview/871891981

[26] D Tamaki, Cellular stratified spaces, I: Face categories and classifying spaces, preprint (2011) arXiv:1106.3772

[27] S Zanos, Méthodes de scindements homologiques en topologie et en géométrie, $\mathrm{PhD}$ thesis, Université Lille 1 (2009)

SK: Department of Mathematics, American University of Sharjah Sharjah, United Arab Emirates

IS: Ecole nationale supérieure d'ingénieurs de Tunis, Université de Tunis 05, Avenue Taha Hussein, 1008 Montfleury, Tunisia

IS: Laboratoire LATAO, Faculté des sciences de Tunis, Université de Tunis-El Manar sadok.kallel@math.univ-lille1.fr, ines.saihi@esstt.rnu.tn

Received: 22 September 2015 Revised: 15 January 2016 